MATHEMATICS OF COMPUTATION

Volume 74, Number 251, Pages 1257-1280

S 0025-5718(04)01719-3

Article electronically published on December 8, 2004

\title{
APPROXIMATIONS OF A GINZBURG-LANDAU MODEL FOR SUPERCONDUCTING HOLLOW SPHERES BASED ON SPHERICAL CENTROIDAL VORONOI TESSELLATIONS
}

\author{
QIANG DU AND LILI JU
}

\begin{abstract}
In this paper the numerical approximations of the GinzburgLandau model for a superconducting hollow spheres are constructed using a gauge invariant discretization on spherical centroidal Voronoi tessellations. A reduced model equation is used on the surface of the sphere which is valid in the thin spherical shell limit. We present the numerical algorithms and their theoretical convergence as well as interesting numerical results on the vortex configurations. Properties of the spherical centroidal Voronoi tessellations are also utilized to provide a high resolution scheme for computing the supercurrent and the induced magnetic field.
\end{abstract}

\section{INTRODUCTION}

The quantized vortex phenomena are well-known signatures of superfluidity. The macroscopic model of Ginzburg and Landau [15, 33. has been widely used to describe the vortex state in both low-temperature and high-temperature superconductors. The nucleation of quantized vortices in superconductors due to the applied magnetic field has been rigorously established and extensively simulated based on the phenomenological Ginzburg-Landau equations; see, for example, 2, 3, 4, 16, 19 21, 22, 26] and the references cited therein. The numerical approximations of Ginzburg-Landau type models have also been studied, including the popular finite difference methods [12, 23] and finite element methods [8, 15, 16]. Based on a planar Voronoi-Delaunay grid, covolume techniques have also been analyzed, and they provide gauge invariant approximations to the Ginzburg-Landau model 20]. It is clear that methods based on unstructured grids, for example, the finite element and finite volume methods become handy when we simulate superconducting samples of various geometric forms.

The geometry we focus on in this paper is a thin spherical superconducting shell. In the physics literature, various studies on superconducting samples with spherical geometry have been made, ranging from superconducting balls to hollow spheres [9] 11, 34, 35. Superconducting hollow spheres play important roles in the technological applications of superconductivity, such as in the design of the superconducting gravimeters [29] and the Gravity Probe B gyroscopes developed

Received by the editor July 13, 2003 and, in revised form, January 5, 2004.

2000 Mathematics Subject Classification. Primary 65N15, 65N99; Secondary 82D55.

Key words and phrases. Ginzburg-Landau model of superconductivity, finite volume, gauge invariance, convergence, spherical centroidal Voronoi tessellations.

This work is supported in part by NSF-DMS 0196522 and NSF-ITR 0205232. 
at Stanford [25] for testing the theory of general relativity. In light of the latest development in superconducting nano-clusters [31, 32, spherical geometries and thus the vortex structures related to spherical geometries may be of great interest for future superconductivity research.

Taking advantage of the thin structure, a reduction of the full three-dimensional Ginzburg-Landau model can be made which still captures many basic features of the vortex state 6 . The resulting model becomes a simplified equation for the order parameter defined on the sphere surface, similar to the case of a thin film [3, 5, 7. To simulate the simplified equation, a well-designed approximation scheme is needed and a high-quality spherical grid is also desirable as it is well known that a perfect uniformly distributed grid does not exist on the sphere for all levels of resolution.

Recently, we have made numerous studies on generating high-quality, almostuniform spherical tessellations that we call spherical centroidal Voronoi tessellations [14. We have also studied the application of finite volume approximation of convection diffusion equations on such grids [14, 17]. In this paper, we extend the finite volume methods to construct a gauge invariant approximation of the reduced Ginzburg-Landau (G-L) model. The novelty of the extension lies in its implementation on a spherical centroidal Voronoi tessellation (SCVT) which provides a higher order resolution of the physical variables than on a conventional spherical Voronoi-Delaunay grid. Such a claim results from the optimality of SCVT on the sphere: the SCVT gives a high quality mesh for both the numerical solution and their gradient recovery; it also leads to optimal quadratures for numerical integrations on the sphere. As illustrated in the paper, the Ginzburg-Landau model on the sphere is a typical nonlinear system that provides a natural setting to utilize these optimal spherical centroidal Voronoi tessellations. While a brief convergence theory of our approximations is provided, the focus of the paper is on the discussion of the numerical algorithm, some of its interesting properties, its implementation with respect to the spherical centroidal Voronoi grids, and the effective evaluation of various physically relevant quantities. We also present numerical simulations of the vortex structure on the spherical shell generated by a constant applied magnetic field and obtain the second order of convergence numerically.

The paper is organized as follows. In Section 2 we describe the basic GinzburgLandau theory and the reduced model for the spherical shell. In Section 3 finite volume approximations are discussed. The SCVT grids are briefly discussed in Section 4. In Section 5 some theoretical issues concerning the convergence of the numerical approximations are examined. In Section 6 we discuss how to utilize the SCVT to compute the physical variables. Some numerical examples are given in Section 7, and final conclusions are given in Section 8.

\section{The Ginzburg-Landau model For Superconductivity}

Let $\Omega \subset \mathbb{R}^{d}(d=3)$ be the region occupied by the superconducting sample. The primary variables used in the equilibrium G-L model are the complex scalarvalued order parameter $\psi$ and the real vector-valued magnetic potential $\mathbf{A}$ (for time-dependent models, the real scalar-valued electric potential $\bar{\Phi}$ is also needed). 
According to Ginzburg and Landau [15, 33], in a nondimensionalized form, the conventional Gibbs free energy is given by

$$
\mathcal{G}(\psi, \mathbf{A})=\int_{\Omega}\left(\frac{1}{2}\left|\left(\frac{i}{\kappa} \nabla+\mathbf{A}\right) \psi\right|^{2}+\frac{1}{4}\left(1-|\psi|^{2}\right)^{2}+\frac{1}{2} \int_{\mathbb{R}^{d}}|\operatorname{curl} \mathbf{A}-\vec{H}|^{2}\right) d \Omega,
$$

where $\kappa$, the Ginzburg-Landau parameter, is a material constant and $\vec{H}$ is a given applied magnetic field.

The minimizers of the free energy functional satisfy the G-L equations

$$
\begin{gathered}
\left(\frac{i}{\kappa} \nabla+\mathbf{A}\right)^{2} \psi-\psi+|\psi|^{2} \psi=0 \quad \text { in } \Omega, \\
\text { curl curl } \mathbf{A}+\frac{i}{2 \kappa}\left(\psi^{*} \nabla \psi-\psi \nabla \psi^{*}\right)+|\psi|^{2} \mathbf{A}=0 \quad \text { in } \Omega, \\
\operatorname{curl} \operatorname{curl} \mathbf{A}=0 \quad \text { in } \mathbb{R}^{d} \backslash \Omega,
\end{gathered}
$$

with curl $\mathbf{A}=\vec{H}$ at infinity. Solutions of the G-L equations satisfy the gauge invariance property. Assume that $\vec{H}=H \vec{z}$ is a constant field in the $z$-direction with strength $H$, and let $\mathbf{A}_{0}=\mathbf{A}_{0}(\mathbf{x})$ be given by

$$
\mathbf{A}_{0}(x, y, z)=\frac{H}{2}(y,-x, 0)^{T} .
$$

A popular gauge choice is given by $\operatorname{div} \mathbf{A}=0$ and $\mathbf{A} \rightarrow \mathbf{A}_{0}$ at infinity.

It turns out a reduction of the model is possible when the domain $\Omega$ is made of a very thin structure [3, 5]. For example, in the case of a thin shell under constant field in the $z$-direction, with $\delta a(\mathbf{x})$ being the thickness function and $\delta$ being a small constant, the solution of the above equations can be approximated, to the leading order of $\delta$, by $\left(\psi, \mathbf{A}_{0}\right)$ where the function $\psi$ is defined on the sphere surface $\mathbb{S}^{2}=\{\mathbf{x} \mid\|\mathbf{x}\|=r>0\}[6]$, in the same spirit as of the thin film.

Denote by $\nabla_{s}$ the surface tangential gradient 24 on the sphere defined as

$$
\nabla_{s}=\left(\nabla_{s, 1}, \nabla_{s, 2}, \nabla_{s, 3}\right)=\nabla-\left(\nabla \cdot \overrightarrow{\mathbf{n}}_{\mathbf{x}}\right) \overrightarrow{\mathbf{n}}_{\mathbf{x}}
$$

where $\nabla$ is the standard gradient operator and $\overrightarrow{\mathbf{n}}_{\mathbf{x}}$ is the outer normal vector toward $\mathbb{S}^{2}$ at $\mathbf{x}$, by $\mathbf{A}_{0 s}$ the tangential projection of $\mathbf{A}_{0}$ defined as

$$
\mathbf{A}_{0 s}=\mathbf{A}_{0}-\left(\mathbf{A}_{0} \cdot \overrightarrow{\mathbf{n}}_{\mathbf{x}}\right) \overrightarrow{\mathbf{n}}_{\mathbf{x}} .
$$

After a rescaling, the reduced equation satisfied by $\psi$ then becomes

$$
-\left(\nabla_{s}-i \mathbf{A}_{0 s}\right) a(\mathbf{x})\left(\nabla_{s}-i \mathbf{A}_{0 s}\right) \psi+\frac{1}{\epsilon^{2}} \psi\left(|\psi|^{2}-1\right)=0, \quad \text { on } \mathbb{S}^{2},
$$

where $a>0$ is a function measuring the relative thickness of the three-dimensional thin spherical shell. The parameter $\epsilon$ can be interpreted as the effective coherence length which depends on $\kappa$ for the thin shell; see [5] for details. For simplicity, here we let $a(\mathbf{x})=1$; in other words, we assume that the spherical shell has uniform thickness.

It is easy to see that the solutions of equation (2.1) are the critical points of the energy functional

$$
\mathcal{F}(\psi)=\int_{\mathbb{S}^{2}}\left(\left|\left(\nabla_{s}-i \mathbf{A}_{0 s}\right) \psi\right|^{2}+\frac{1}{2 \epsilon^{2}}\left(1-|\psi|^{2}\right)^{2}\right) d S .
$$


It is in our interest here to first compute for the ground state of the energy, and then to calculate all solution branches for (2.1) to obtain an accurate bifurcation diagram, similar to the study made in [2].

The gauge invariance of the functional $\mathcal{F}$, and thus, the gauge invariance of the solution space for the Ginzburg-Landau equations, is understood as $\mathcal{F}$ being invariant under the transformation: for any smoothly defined real-valued scalar function $\eta=\eta(\mathbf{x})$,

$$
\left(\mathbf{A}_{0 s}, \psi\right) \rightarrow\left(\mathbf{A}_{0 s}+\nabla_{s} \eta, \psi \exp (i \eta)\right) .
$$

Another feature related to the gauge invariance in the solution space, in the spherical setting, is the rotational group invariance in the independent variables with respect to the $z$ axis. In fact, if $\psi=\psi(\mathbf{x})$ is a solution of equation (2.1), and $T: \mathbb{S}^{2} \rightarrow \mathbb{S}^{2}$ is a plane rotation

$$
T=\left(\begin{array}{ccc}
\cos \alpha & \sin \alpha & 0 \\
-\sin \alpha & \cos \alpha & 0 \\
0 & 0 & 1
\end{array}\right),
$$

where $\alpha$ is the angle of rotation, then $\hat{\psi}=\hat{\psi}(\mathbf{x})$ defined by

$$
\hat{\psi}(\mathbf{x})=\psi(T \mathbf{x}) \exp \left(i x^{2} \sin \alpha / 2+i x y(1-\cos \alpha)+i y^{2} \sin \alpha / 2+i \beta\right)
$$

for $\mathbf{x}=(x, y, z)^{T} \in \mathbb{S}^{2}$, is also a solution for an arbitrary real scalar constant $\beta$. The invariance with respect to $\beta$ gives the $U(1)$ symmetry of the solution space.

A consequence of the gauge and rotation invariance is that rigorous numerical analysis is best applied in a properly defined quotient space. Such a point is to be further addressed in a future work.

\section{Finite volume approximations}

Denote by $d(\mathbf{x}, \mathbf{y})$ the geodesic distance between $\mathbf{x}$ and $\mathbf{y}$ on $\mathbb{S}^{2}$; i.e.,

$$
d(\mathbf{x}, \mathbf{y})=r\left[\arccos \left(\frac{\mathbf{x} \cdot \mathbf{y}}{r^{2}}\right)\right] \quad \forall \mathbf{x}, \mathbf{y} \in \mathbb{S}^{2} .
$$

Given a set of distinct points $\left\{\mathbf{x}_{j}\right\}_{j=1}^{n} \subset \mathbb{S}^{2}$, we can define for each point $\mathbf{x}_{j}$, $j=1, \ldots, n$, the corresponding Voronoi region $V_{j}, j=1, \ldots, n$, by

$$
V_{j}=\left\{\mathbf{y} \in \mathbb{S}^{2} \quad \mid \quad d\left(\mathbf{x}_{j}, \mathbf{y}\right)<d\left(\mathbf{x}_{k}, \mathbf{y}\right) \quad \text { for } \quad k=1, \ldots, n \text { and } k \neq j\right\} .
$$

Clearly, we have $\mathbf{x}_{j} \in V_{j}$, and $\left\{V_{j}\right\}_{j=1}^{n}$ forms a tessellation of $\mathbb{S}^{2}$. We refer to $\left\{V_{j}\right\}_{j=1}^{n}$ as the Voronoi tessellation or Voronoi diagram of $\mathbb{S}^{2}$ associated with the point set $\left\{\mathbf{x}_{j}\right\}_{j=1}^{n}$. We call $\mathbf{x}_{j}$ a generator, and a subdomain $V_{j} \subset \mathbb{S}^{2}$ is referred to as the Voronoi region or Voronoi cell corresponding to the generator $\mathbf{x}_{j}$. It is easy to see that each Voronoi cell $V_{j}$ is an open convex spherical polygon on $\mathbb{S}^{2}$; i.e., its sides are geodesic arcs. It is also well known that the dual tessellation to a Voronoi tessellation of $\mathbb{S}^{2}$ consists of spherical triangles. The dual tessellations [28] are referred to as Delaunay triangulations and are very popular computational meshes on the sphere, similar to the planar cases.

Given a spherical Voronoi-Delaunay mesh $\mathcal{W}=\left\{\mathbf{x}_{j}, V_{j}\right\}_{j=1}^{n}$ (see Figure 1), define

$$
m(A)=\left\{\begin{array}{l}
\text { the area of } A \text { if it is a nonempty subdomain of } \mathbb{S}^{2}, \\
\text { the length of } A \text { if it is a geodesic arc on } \mathbb{S}^{2} .
\end{array}\right.
$$



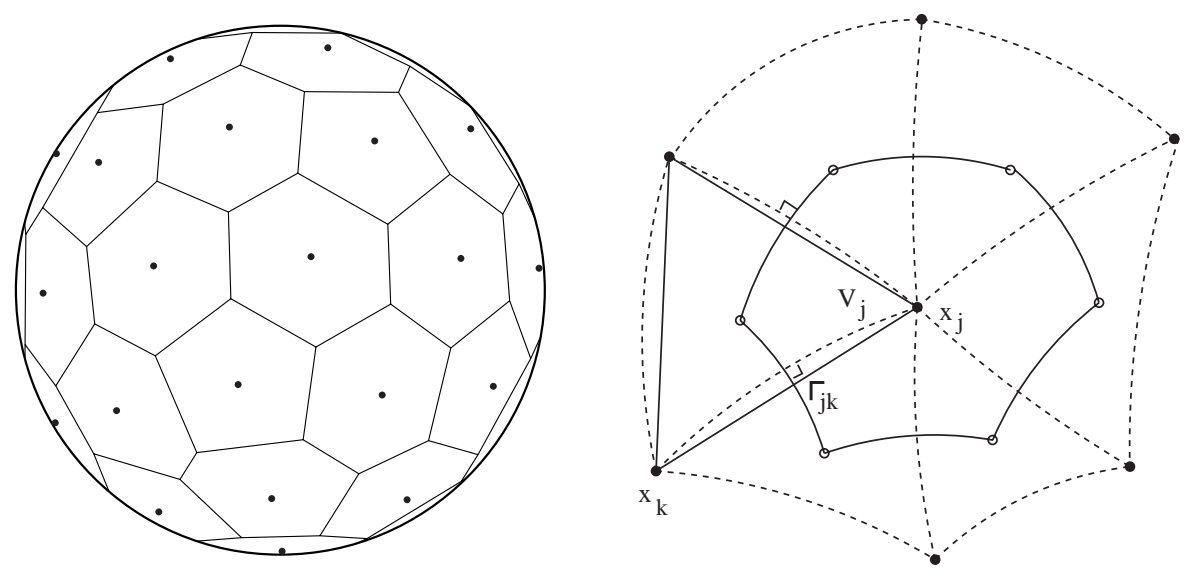

Figure 1. Left: a spherical Voronoi tessellation; right: a single Voronoi region and its dual triangles.

Then, for $\vec{\psi}^{h}=\left(\psi_{1}, \psi_{2}, \ldots, \psi_{n}\right)$, the Ginzburg-Landau functional is discretized as

$$
\begin{aligned}
\mathcal{F}^{h}\left(\vec{\psi}^{h}\right)=\frac{1}{2 \epsilon^{2}} \sum_{j=1}^{n}\left\{m\left(V_{j}\right)\left(1-\left|\psi_{j}\right|^{2}\right)^{2}\right\} \\
+\frac{1}{2} \sum_{j=1}^{n} \sum_{k \in \chi_{j}}\left\{\frac{m\left(\Gamma_{j k}\right)}{\left\|\mathbf{x}_{j}-\mathbf{x}_{k}\right\|}\left|\psi_{k} \exp \left(-i c_{j k}\right)-\psi_{j}\right|^{2}\right\},
\end{aligned}
$$

where

$$
c_{j k}=\int_{\widetilde{\mathbf{x}_{k} \mathbf{x}_{j}}} \mathbf{A}_{0 s}(\mathbf{x}) \cdot \overrightarrow{\mathbf{t}}_{j k} d \ell=\left(\int_{\widetilde{\mathbf{x}_{k} \mathbf{x}_{j}}} \mathbf{A}_{0 s}(\mathbf{x}) d \ell\right) \cdot \overrightarrow{\mathbf{t}}_{j k},
$$

where $\overrightarrow{\mathbf{t}}_{j k}$ denotes the unit tangent field along the geodesic arc $\widetilde{\mathbf{x}_{j} \mathbf{x}_{k}}$ at the middle point of $\widetilde{\mathbf{x}_{j} \mathbf{x}_{k}}$. In fact, due to symmetry, we have

$$
\overrightarrow{\mathbf{t}}_{j k}=\frac{\mathbf{x}_{k}-\mathbf{x}_{j}}{\left\|\mathbf{x}_{k}-\mathbf{x}_{j}\right\|} .
$$

For any $j$, the index set $\chi_{j}$ denotes the indices of all vertices which are adjacent to the vertex $\mathbf{x}_{j}$. We also assume that for two adjacent vertices $\mathbf{x}_{k}$ and $\mathbf{x}_{j}$, the common boundary $\Gamma_{j k}$ of their Voronoi regions (part of their bisector, i.e., points on the sphere having equal distance to $\mathbf{x}_{k}, \mathbf{x}_{j}$ ) has a nonzero measure $m\left(\Gamma_{j k}\right)$.

The choice of the above discrete energy is made in order to preserve several properties shared by the continuous energy functional at the discrete level: one being the validity of the maximum principle, which states that the magnitude of the order parameter should not exceed the unit (constant 1); another being the gauge invariance in the discrete sense. Detailed discussions on gauge invariant approximations to the full Ginzburg-Landau models can be found in [12, 20. As the original continuous model enjoys gauge invariance, it is desirable that such a property still holds at the discrete level. Moreover, the gauge invariance gives one the freedom to choose the most convenient gauge to work with and makes the gauge fixing much easier to implement numerically [12. For the reduced model given above, the discrete gauge transformation may be defined by

$$
\left(\mathbf{A}_{0 s},\left\{\psi_{j}\right\}_{j=1}^{n}\right) \rightarrow\left(\mathbf{A}_{0 s}+\nabla_{s} \eta(\mathbf{x}),\left\{\psi_{j} \exp \left(i \eta\left(\mathbf{x}_{j}\right)\right)\right\}_{j=1}^{n}\right) .
$$


It is easy to see that the discrete energy functional is invariant under the discrete gauge transformation. The rotational invariance can be understood in the sense that, for an arbitrary mesh, a plane rotation would lead to a new mesh and a discrete solution on the new mesh can be obtained from a solution in the old mesh. Notice that for a given mesh (such as the SCVT grid discussed here), the discrete solution is possibly rotation invariant only for a finite rotation group with a cardinality independent of the mesh size. Thus, we do not have to worry about the rotation invariance (except the $U(1)$ symmetry given by a constant phase change) of the discrete solution space. The $U(1)$ symmetry can be fixed by explicitly assigning the phase variable at a particular point (typically chosen to be located on the equator where the solution has nonzero magnitude).

Let $\vec{\psi}^{h}$ represent the vector with components $\left\{\psi_{j}\right\}$, and define

$$
F_{j k}\left(\vec{\psi}^{h}\right)=-m\left(\Gamma_{j k}\right) \frac{\psi_{k} \exp \left(-i c_{j k}\right)-\psi_{j}}{\left\|\mathbf{x}_{j}-\mathbf{x}_{k}\right\|} .
$$

Then the Euler-Lagrange equation gives

$$
\frac{1}{m\left(V_{j}\right)} \sum_{k \in \chi_{j}} F_{j k}+\frac{1}{\epsilon^{2}} \psi_{j}\left(\left|\psi_{j}\right|^{2}-1\right)=0 \quad \text { for } j=1, \ldots, n .
$$

The gauge invariance of the discrete free energy functional naturally implies that the above finite volume scheme is also gauge invariant. Gauge invariant finite difference methods have been widely used in the physics literature [1, 23]. Though uniform cartesian grids do not exist for the sphere, the invariant approximation given above provides a natural extension of the standard gauge invariant finite difference methods based on spherical geometry.

\section{Mesh Regularity AND the SCVT}

To ensure good approximation properties, some mesh regularity assumptions on the underlying grids are needed.

Given a Voronoi mesh $\mathcal{W}=\left\{\mathbf{x}_{j}, V_{j}\right\}_{j=1}^{n}$, we define the mesh norm $h$ by

$$
h=\max _{j=1, \ldots, n} h_{j}, \quad \text { where } \quad h_{j}=\max _{\mathbf{y} \in V_{j}} d\left(\mathbf{x}_{j}, \mathbf{y}\right) .
$$

Thus, $h_{j}$ gives the maximum geodesic distance between the particular generator $\mathbf{x}_{j}$ and the points in its associated cell $V_{j}$, and $h$ gives the maximum geodesic distance between any generator and the points in its associated cell. We then define the mesh regularity norm $\sigma$ [14] by

$$
\sigma=\min _{j=1, \ldots, n} \min _{k \in \chi_{j}} \frac{d\left(\mathbf{x}_{j}, \mathbf{x}_{k}\right)}{2 h_{j}} .
$$

$\sigma$ can be used as a measure of the uniformity of a mesh; the larger the value of $\sigma$, the more uniform the mesh. In addition, the value of $\sigma$ provides us a measure of the mesh regularity; i.e., the local uniformity of a mesh. We will refer to $\mathcal{W}$ as regular if $\sigma$ is uniformly bounded above zero for small $h$ (large $n$ ).

An optimal spherical mesh that gives good mesh regularity is the one given by the SCVT [14. The SCVT is a spherical Voronoi tessellation with special distributions of their Voronoi generators.

Given a density function $\rho$ defined on $\mathbb{S}^{2}$, for any spherical region $V \subset \mathbb{S}^{2}$, the spherical mass centroid $\mathbf{x}^{c}$ of $V$ on $\mathbb{S}^{2}$ is given by the minimizer of

$$
\min _{\mathbf{x} \in V} \mathbf{e}(\mathbf{x}), \quad \text { where } \quad \mathbf{e}(\mathbf{x})=\int_{V} \rho(\mathbf{y})\|\mathbf{y}-\mathbf{x}\|^{2} d s(\mathbf{y}),
$$


where $d s(\mathbf{y})$ denotes the area element at $\mathbf{y} \in V \subset \mathbb{S}^{2}$. As in [14, a Voronoi tessellation of $\mathbb{S}^{2}$ is called a spherical centroidal Voronoi tessellation if and only if the points $\left\{\mathbf{x}_{j}\right\}_{j=1}^{n}$ which serve as the generators of the associated spherical Voronoi tessellation $\left\{V_{j}\right\}_{j=1}^{n}$ are also the spherical mass centroids of those Voronoi regions. The SCVTs are special cases on the sphere of the constrained CVTs for general surfaces [13. It can be shown [13, that a properly defined energy is minimized only when $\left\{\mathbf{x}_{j}, V_{j}\right\}_{j=1}^{n}$ forms a SCVT.

Consequently, spherical centroidal Voronoi meshes have many good geometric properties; see [14]. In particular, for large $n$, it gives very good mesh regularity, and, when applied to the finite volume approximations of linear convection diffusion equations, it leads to high resolution of both the solution and their gradients [17]. Examples of the SCVT grids are given later in the paper.

In our study here, we only take the special case of a constant density $\rho=1$. In such a case, the SCVT generators are uniformly distributed.

\section{AnAlysis of the Finite volume Methods}

We define the standard Sobolev space on $\mathbb{S}^{2}$ (a compact, two-dimensional Riemannian manifold) [24, 14, 17] such as

$$
W^{m, p}\left(\mathbb{S}^{2}\right)=\left\{\left.\phi(\mathbf{x}) \quad\left|\quad \int_{\mathbb{S}^{2}}\right| \nabla_{s}^{\alpha} \phi\right|^{p} d s(\mathbf{x})<\infty, \quad \text { for } 0 \leq|\alpha| \leq m\right\},
$$

where $\alpha=\left(\alpha_{1}, \alpha_{2}, \alpha_{3}\right), \nabla_{s}^{\alpha}=\nabla_{s, 1}^{\alpha_{1}} \nabla_{s, 2}^{\alpha_{2}} \nabla_{s, 3}^{\alpha_{3}}$, and $|\alpha|=\alpha_{1}+\alpha_{2}+\alpha_{3}$. Also,

$$
\|\phi\|_{m, p, \mathbb{S}^{2}}= \begin{cases}\left(\sum_{0 \leq|\alpha| \leq m}\left\|\nabla_{s}^{\alpha} \phi\right\|_{0, p, \mathbb{S}^{2}}^{p}\right)^{1 / p} & \text { for } 1 \leq p<\infty \\ \max _{0 \leq|\alpha| \leq m}\left\|\nabla_{s}^{\alpha} \phi\right\|_{0, \infty, \mathbb{S}^{2}} & \text { for } p=\infty\end{cases}
$$

For the case $p=2$, we let $\mathcal{H}^{m}\left(\mathbb{S}^{2}\right)=W^{m, 2}\left(\mathbb{S}^{2}\right)$ and $\|\phi\|_{m, \mathbb{S}^{2}}=\|\phi\|_{m, 2, \mathbb{S}^{2}}$.

If $\mathbf{x}_{j_{1}}, \mathbf{x}_{j_{2}}$, and $\mathbf{x}_{j_{3}}$ are neighbors for each other in $\mathcal{W}$, we denote by $\tilde{\tau}_{j k l}$ the spherical triangle determined by $\mathbf{x}_{j_{1}}, \mathbf{x}_{j_{2}}$, and $\mathbf{x}_{j_{3}}$, and by $\tau_{j_{1} j_{2} j_{3}}$ the corresponding planar triangle. We use the notation $\Sigma=\left\{V_{j}\right\}_{j=1}^{n}, \tilde{\mathcal{T}}=\left\{\tilde{\tau}_{j_{1} j_{2} j_{3}}\right\}$, and $\mathcal{T}=\left\{\tau_{j_{1} j_{2} j_{3}}\right\}$. It is easy to see that

$$
\tilde{\tau}_{j_{1} j_{2} j_{3}}=\left\{\mathcal{P}(\mathbf{y})=\mathbf{y} /\|\mathbf{y}\| \mid \mathbf{y} \in \tau_{j_{1} j_{2} j_{3}}\right\}=\mathcal{P}\left(\tau_{j_{1} j_{2} j_{3}}\right)
$$

and $\tilde{\mathcal{T}}$ is the corresponding spherical Delaunay triangulation of $\mathbb{S}^{2}$ associated with the generators $\left\{\mathbf{x}_{j}\right\}_{j=1}^{n}$. We can also view $\mathcal{P}$ as a one-to-one smooth function that maps $\mathbb{S}^{*}=\bigcup_{\tau_{j_{1} j_{2} j_{3} \in \mathcal{T}}} \tau_{j_{1} j_{2} j_{3}}$ to $\mathbb{S}^{2}=\bigcup_{\tilde{\tau}_{j_{1} j_{2} j_{3}} \in \tilde{\mathcal{T}}} \tilde{\tau}_{j_{1} j_{2} j_{3}}$. Clearly, $\mathbb{S}^{*} \cap \mathbb{S}^{2}=\left\{\mathbf{x}_{i}\right\}_{i=1}^{n}$.

From now on, we assume the mesh regularity on the spherical Voronoi-Delaunay mesh used in the discretization (3.1), which is automatically satisfied by the SCVT grid with sufficiently many generators. Moreover, for such a SCVT grid, all angles of the corresponding planar triangles in $\mathcal{T}$ are acute for large enough $n$.

For any $\mathbf{x} \in \mathbb{S}^{2}$ and $\mathbf{x}_{1}, \mathbf{x}_{2} \in \tilde{\tau}_{j_{1} j_{2} j_{3}}$, we have

$$
\left\{\begin{array}{l}
\left\|\mathbf{x}-\mathcal{P}^{-1}(\mathbf{x})\right\| \leq c h^{2} \\
\left(1-c h^{2}\right) d\left(\mathbf{x}_{1}, \mathbf{x}_{2}\right) \leq\left\|\mathcal{P}^{-1}\left(\mathbf{x}_{1}\right)-\mathcal{P}^{-1}\left(\mathbf{x}_{2}\right)\right\| \leq\left(1+c h^{2}\right) d\left(\mathbf{x}_{1}, \mathbf{x}_{2}\right), \\
m\left(\tau_{j_{1} j_{2} j_{3}}\right) \leq m\left(\tilde{\tau}_{j_{1} j_{2} j_{3}}\right) \leq\left(1+c h^{2}\right) m\left(\tau_{j_{1} j_{2} j_{3}}\right),
\end{array}\right.
$$

where $m\left(\tau_{j_{1} j_{2} j_{3}}\right)$ denotes the area of the triangle $\tau_{j_{1} j_{2} j_{3}}$ and $c$ represents a generic constant that is independent of $h$ for $h$ small. 
Given $\vec{\phi}^{h}=\left(\phi_{1}, \phi_{2}, \ldots, \phi_{n}\right)$, we call $\phi^{h, L}$ a piecewise linear function on $\mathbb{S}^{*}$ that interpolates $\vec{\phi}^{h}$ at all the vertices $\left\{\mathbf{x}_{j}\right\}_{j=1}^{n}$ if and only if

$$
\phi^{h, L}(\mathbf{y})=\lambda_{j_{1}} \phi_{j_{1}}+\lambda_{j_{2}} \phi_{j_{2}}+\lambda_{j_{3}} \phi_{j_{3}} \quad \forall \mathbf{y} \in \tau_{j_{1} j_{2} j_{3}},
$$

where $\lambda_{j_{1}}, \lambda_{j_{2}}, \lambda_{j_{3}}$ are the barycentric coordinates of $\mathbf{y}$ in the planar triangle $\tau_{j_{1} j_{2} j_{3}}$. Based on $\phi^{h, L}$, we define a special approximate piecewise linear approximation that interpolates $\vec{\phi}^{h}$ at all the vertices on the sphere $\mathbb{S}^{2}$ by

$$
\phi^{h}(\mathbf{x})=\phi^{h, L}(\mathbf{y})=\phi^{h, L}\left(\mathcal{P}^{-1}(\mathbf{x})\right), \quad \mathbf{x} \in \mathbb{S}^{2} .
$$

For the properties of such an interpolation on $\mathbb{S}^{2}$, see $[14,17$. It is easy to find that $\phi^{h} \in \mathcal{H}^{1}\left(\mathbb{S}^{2}\right)$.

Let $\nabla_{*}$ be the tangential surface gradient operator on $\mathbb{S}^{*}$, i.e., for any function $\xi$ defined on $\mathbb{S}^{*}$,

$\nabla_{*} \xi(\mathbf{y})=\left(\nabla_{*, 1}, \nabla_{*, 2}, \nabla_{*, 3}\right) \xi(\mathbf{y})=\nabla \xi(\mathbf{y})-\left(\nabla \xi(\mathbf{y}) \cdot \overrightarrow{\mathbf{n}}_{\tau_{j_{1} j_{2} j_{3}}}\right) \overrightarrow{\mathbf{n}}_{\tau_{j_{1} j_{2} j_{3}}} \quad \forall \mathbf{y} \in \tau_{j_{1} j_{2} j_{3}}$, where $\overrightarrow{\mathbf{n}}_{\tau_{j_{1} j_{2} j_{3}}}$ is the unit outer normal vector to $\tau_{j_{1} j_{2} j_{3}}$. In fact, the operator $\nabla_{*}$ is locally equivalent to the standard two-dimensional gradient operator in each planar triangle $\tau_{j_{1} j_{2} j_{3}}$; consequently, we know that for any $\mathbf{y}_{1}, \mathbf{y}_{2} \in \tau_{j_{1} j_{2} j_{3}}$,

$$
\nabla_{*} \phi^{h, L}\left(\mathbf{y}_{1}\right)=\nabla_{*} \phi^{h, L}\left(\mathbf{y}_{2}\right)
$$

since $\phi^{h, L}$ is linear on $\tau_{j_{1} j_{2} j_{3}}$.

For convenience, we define $\mathbf{A}_{0 *}$ as the piecewise projection of $\mathbf{A}_{0 s}$ onto $\mathbb{S}^{*}$; that is,

in each $\tau_{j_{1} j_{2} j_{3}}$. Clearly,

$$
\mathbf{A}_{0 *}(\mathbf{y})=\mathbf{A}_{0 s}(\mathcal{P}(\mathbf{y}))-\left(\mathbf{A}_{0 s}(\mathcal{P}(\mathbf{y})) \cdot \overrightarrow{\mathbf{n}}_{\tau_{j_{1} j_{2} j_{3}}}\right) \overrightarrow{\mathbf{n}}_{\tau_{j_{1} j_{2} j_{3}}},
$$

$$
\begin{gathered}
\left|\mathbf{A}_{0 *}(\mathbf{y})\right| \leq\left|\mathbf{A}_{0 s}(\mathcal{P}(\mathbf{y}))\right|, \\
\left|\mathbf{A}_{0 s}(\mathcal{P}(\mathbf{y}))-\mathbf{A}_{0 *}(\mathbf{y})\right| \leq c h\left|\mathbf{A}_{0 s}(\mathcal{P}(\mathbf{y}))\right| .
\end{gathered}
$$

We define a modified energy functional: for $\phi$ with $\|\phi\|_{1, \mathbb{S}^{*}}<\infty$,

$$
\mathcal{F}^{L}(\phi)=\sum_{\tau_{j_{1} j_{2} j_{3}} \in \mathcal{T}} \int_{\tau_{j_{1} j_{2} j_{3}}}\left(\left|\left(\nabla_{*}-i \mathbf{A}_{0 *}\right) \phi\right|^{2}+\frac{1}{2 \epsilon^{2}}\left(1-|\phi|^{2}\right)^{2}\right) d \tau .
$$

Here, we let $\|\phi\|_{1, \mathbb{S}^{*}}^{2}=\sum_{\tau_{j_{1} j_{2} j_{3}} \in \mathcal{T}}\|\phi\|_{1, \tau_{j_{1} j_{2} j_{3}}}^{2}$.

It is also easy to find that for any $\mathbf{y} \in \tau_{j_{1} j_{2} j_{3}}$ and $\mathbf{x}=\mathcal{P}(\mathbf{y}) \in \mathbb{S}^{2}$, we have

$$
\overrightarrow{\mathbf{n}}_{\mathbf{x}} \cdot \overrightarrow{\mathbf{n}}_{\tau_{j_{1} j_{2} j_{3}}} \geq 1-c h^{2} .
$$

Using (5.1), (5.2), and similar analyses in [14, 17], we have that for any $\mathbf{x} \in$ $\tilde{\tau}_{j_{1} j_{2} j_{3}}, \mathbf{y}=\mathcal{P}^{-1}(\mathbf{x}) \in \tau_{j_{1} j_{2} j_{3}}$

$$
\left|\nabla_{s} \phi^{h}(\mathbf{x})-\nabla_{*} \phi^{h, L}(\mathbf{y})\right| \leq c h\left|\nabla_{s} \phi^{h}(\mathbf{x})\right|, \quad j=j_{1}, j_{2}, j_{3},
$$

and

$$
\left|\nabla_{s} \phi^{h}(\mathbf{x})-\nabla_{*} \phi^{h, L}(\mathbf{y})\right| \leq c h\left|\nabla_{*} \phi^{h, L}(\mathbf{y})\right|, \quad j=j_{1}, j_{2}, j_{3},
$$

for some constant $c>0$. Consequently, let $\phi^{h}$ be an approximate piecewise linear function in $\mathcal{H}^{1}\left(\mathbb{S}^{2}\right)$ corresponding to the spherical triangulation $\tilde{\mathcal{T}}$. Then for $h$ small,

$$
\left(1-c h^{2}\right)\left\|\phi^{h, L}\right\|_{1, \mathbb{S}^{*}}^{2} \leq\left\|\phi^{h}\right\|_{1, \mathbb{S}^{2}}^{2} \leq\left(1+c h^{2}\right)\left\|\phi^{h, L}\right\|_{1, \mathbb{S}^{*}}^{2}
$$

for some constant $c>0$ independent of $h$. 
First, we have the following maximum principle.

Lemma 5.1. If $\vec{\psi}^{h}=\left(\psi_{1}, \psi_{2}, \ldots, \psi_{n}\right)$ is a minimizer of $\mathcal{F}^{h}$ of (3.1), then $\left|\psi_{j}\right| \leq$ 1 for any $j$.

The proof follows the same line of argument as that given in [12, 20] for the planar case.

Given an approximate piecewise linear approximation $\phi^{h}$ with respect to the spherical triangulation $\tilde{\mathcal{T}}$ associated with the spherical Voronoi tessellation $\Sigma$, and $\phi^{h}$ interpolates data $\vec{\phi}^{h} \in \mathbb{C}^{\mathrm{APN}}$ at the vertices in the way defined in (5.3); i.e., $\phi^{h}\left(\mathbf{x}_{j}\right)=\phi_{j}, 1 \leq j \leq n$. Then we have

Lemma 5.2. If $\mathcal{F}^{h}\left(\vec{\phi}^{h}\right)$ is uniformly bounded, then there exists a generic constant $c>0$, independent of $h$, such that

$$
\left\|\phi^{h}\right\|_{1, \mathbb{S}^{2}} \leq c,
$$

and

$$
\left\|\phi^{h, L}\right\|_{1, \mathbb{S}^{*}} \leq c
$$

Proof. From the definition of $\mathcal{F}^{h}$ and the uniform boundedness, we get

$$
\sum_{j}\left|\phi_{j}\right|^{4} m\left(V_{j}\right) \leq c .
$$

By the equivalence of norms in polynomial spaces and scaling argument,

$$
\left\|\phi^{h}\right\|_{0,4, \mathbb{S}^{2}} \leq c
$$

for the piecewise linear function $\psi^{h}$. Then we get

$$
\sum_{k j}\left\{\frac{m\left(\Gamma_{k j}\right)}{\left\|\mathbf{x}_{j}-\mathbf{x}_{k}\right\|}\left|\phi_{k}-\phi_{j}\right|^{2}\right\} \leq c .
$$

Using the equivalence of the norms given in [17, we get the final results for $\phi^{h}$ and $\phi^{h, L}$.

Theorem 5.3. There exists a minimizer $\vec{\psi}^{h}$ of $\mathcal{F}^{h}$. Moreover, $\left|\psi_{j}\right| \leq 1$ for all $j$.

Proof. The functional $\mathcal{F}^{h}$ is obviously continuous and bounded below, and thus has a minimizing sequence $\left\{\vec{\psi}_{n}^{h}\right\}_{n=1}^{\infty}$. Using the previous lemma, $\mathcal{F}^{h}\left(\vec{\psi}_{n}^{h}\right)$ is uniformly bounded above and this implies that $\vec{\psi}_{n}^{h}$ is also uniformly bounded, so that a minimizing sequence must have a convergent subsequence. By continuity, limit of this subsequence is a minimizer of $\mathcal{F}^{h}$. The pointwise bound follows from the maximum principle.

Then, we have the following result.

Lemma 5.4. If $\phi^{h}$ defined above is uniformly bounded independent of $h$ in $\mathcal{H}^{1}\left(\mathbb{S}^{2}\right)$, then

$$
\mathcal{F}\left(\phi^{h}\right)=\mathcal{F}^{L}\left(\phi^{h, L}\right)+o(1) \quad \text { as } h \rightarrow 0
$$

and

$$
\mathcal{F}^{L}\left(\phi^{h, L}\right)=\mathcal{F}^{h}\left(\vec{\phi}^{h}\right)+o(1) \quad \text { as } h \rightarrow 0 .
$$

Consequently,

$$
\mathcal{F}\left(\phi^{h}\right)=\mathcal{F}^{h}\left(\vec{\phi}^{h}\right)+o(1) \quad \text { as } h \rightarrow 0 .
$$


Proof. We note first that $\left\|\phi^{h, L}\right\|_{1, \mathbb{S}^{*}}$ is also uniformly bounded by Lemma 5.2 Using the inequalities (5.1), we have

$$
\begin{aligned}
& \left|\int_{\mathbb{S}^{2}}\left(1-\left|\phi^{h}\right|^{2}\right)^{2} d S-\int_{\mathbb{S}^{*}}\left(1-\left|\phi^{h, L}\right|^{2}\right)^{2} d S^{*}\right| \\
& \leq \sum_{\tilde{\tau}_{j_{1} j_{2} j_{3} \in \tilde{\mathcal{T}}}}\left|\int_{\tilde{\tau}_{j_{1} j_{2} j_{3}}}\left(1-\left|\phi^{h}(\mathbf{x})\right|^{2}\right)^{2} d s(\mathbf{x})-\int_{\tau_{j_{1} j_{2} j_{3}}}\left(1-\left|\phi^{h, L}(\mathbf{y})\right|^{2}\right)^{2} d s^{*}(\mathbf{y})\right| \\
& =\sum_{\tilde{\tau}_{j_{1} j_{2} j_{3}} \in \tilde{\mathcal{T}}} \mid \int_{\tilde{\tau}_{j_{1} j_{2} j_{3}}}\left(1-\left|\phi^{h}(\mathbf{x})\right|^{2}\right)^{2} d s(\mathbf{x}) \\
& \quad-\int_{\tilde{\tau}_{j_{1} j_{2} j_{3}}}\left(1-\left|\phi^{h}(\mathbf{x})\right|^{2}\right)^{2} d s^{*}\left(\mathcal{P}^{-1}(\mathbf{x})\right) \mid \\
& \leq \sum_{\tilde{\tau}_{j_{1} j_{2} j_{3} \in \tilde{\mathcal{T}}} \int_{\tilde{\tau}_{j_{1} j_{2} j_{3}}}\left(1-\left|\phi^{h}(\mathbf{x})\right|^{2}\right)^{2} d s(\mathbf{x})} \leq \operatorname{ch}^{2}\left(m\left(\mathbb{S}^{2}\right)+\left\|\phi^{h}\right\|_{1, \mathbb{S}^{2}}^{4}\right) .
\end{aligned}
$$

Moreover, by the inequalities (5.1), (5.5), (5.8), the norm equivalence, and Sobolev imbedding theorems, we similarly get

$$
\begin{aligned}
& \left.\left|\int_{\tilde{\tau}_{j_{1} j_{2} j_{3}}}\right|\left(i \nabla_{s}+\mathbf{A}_{0 s}\right) \phi^{h}(\mathbf{x})\right|^{2} d s(\mathbf{x})-\int_{\tau_{j_{1} j_{2} j_{3}}}\left|\left(i \nabla_{*}+\mathbf{A}_{0 *}\right) \phi^{h, L}(\mathbf{y})\right|^{2} d s^{*}(\mathbf{y}) \mid \\
& \leq\left|\int_{\tilde{\tau}_{j_{1} j_{2} j_{3}}}\left(\left|\left(i \nabla_{s}+\mathbf{A}_{0 s}\right) \phi^{h}(\mathbf{x})\right|^{2}-\left|\left(i \nabla_{*}+\mathbf{A}_{0 *}\right) \phi^{h, L}\left(\mathcal{P}^{-1}(\mathbf{x})\right)\right|^{2}\right) d s(\mathbf{x})\right| \\
& \quad+\left.\left|\int_{\tau_{j_{1} j_{2} j_{3}}}\right|\left(i \nabla_{*}+\mathbf{A}_{0 *}\right) \phi^{h, L}(\mathbf{y})\right|^{2} d s(\mathcal{P}(\mathbf{y})) \\
& \quad-\int_{\tau_{j_{1} j_{2} j_{3}}}\left|\left(i \nabla_{*}+\mathbf{A}_{0 *}\right) \phi^{h, L}(\mathbf{y})\right|^{2} d s^{*}(\mathbf{y}) \mid \\
& \leq c h^{2}\left(\left\|\phi^{h}\right\|_{1, \tilde{\tau}_{j_{1} j_{2} j_{3}}}^{2}+\left\|\mathbf{A}_{0 s}\right\|_{0,4, \tilde{\tau}_{j_{1} j_{2} j_{3}}}^{2}\left\|\phi^{h}\right\|_{0,4, \tilde{\tau}_{j_{1} j_{2} j_{3}}}^{2}\right) \\
& \quad+c h^{2}\left(\left\|\phi^{h, L}\right\|_{1, \tau_{j_{1} j_{2} j_{3}}}^{2}+\left\|\mathbf{A}_{0 *}\right\|_{0,4, \tau_{j_{1} j_{2} j_{3}}}^{2}\left\|\phi^{h, L}\right\|_{0,4, \tau_{j_{1} j_{2} j_{3}}}^{2}\right) \\
& \leq c h^{2}\left(\left\|\phi^{h}\right\|_{1, \tilde{\tau}_{j_{1} j_{2} j_{3}}}^{2}+\left\|\mathbf{A}_{0 s}\right\|_{1, \tilde{\tau}_{j_{1} j_{2} j_{3}}}^{2}\left\|\phi^{h}\right\|_{1, \tilde{\tau}_{j_{1} j_{2} j_{3}}}^{2}\right) .
\end{aligned}
$$

Then we have

$$
\begin{gathered}
\left.\left|\int_{\mathbb{S}^{2}}\right|\left(i \nabla_{s}+\mathbf{A}_{0 s}\right) \phi^{h}\right|^{2} d S-\int_{\mathbb{S}^{*}}\left|\left(i \nabla_{*}+\mathbf{A}_{s *}\right) \phi^{h, L}\right|^{2} d S^{*} \mid \\
\leq c h^{2}\left(\left\|\phi^{h}\right\|_{1, \mathbb{S}^{2}}^{2}+\left\|\mathbf{A}_{0 s}\right\|_{1, \mathbb{S}^{2}}^{2}\left\|\phi^{h}\right\|_{1, \mathbb{S}^{2}}^{2}\right) .
\end{gathered}
$$

So we get

$$
\left|\mathcal{F}\left(\phi^{h}\right)-\mathcal{F}^{L}\left(\phi^{h, L}\right)\right| \leq c h^{2},
$$

which directly induces (5.9)).

The proof of (5.10) is based on similar consideration given in [20] for the planar case. Using standard approximation results, scaling argument and the norm equivalence in [17] with (5.1), we have for any continuous piecewise polynomial $\bar{\zeta}$ (of degree $\leq 4$ ) defined on $\mathbb{S}^{*}$ with respect to the triangulation $\mathcal{T}$,

$$
\left|\int_{\mathbb{S}^{*}} \bar{\zeta} d S^{*}-\sum_{V_{j_{1}} \in \Sigma} \bar{\zeta}\left(\mathbf{x}_{j_{1}}\right) m\left(V_{j_{1}}\right)\right| \leq c h\|\bar{\zeta}\|_{1,1, \mathbb{S}^{*}},
$$

where $c$ is a generic constant independent of $h$. 
Let $\bar{\zeta}=\frac{1}{2}\left(1-\left|\phi^{h, L}\right|^{2}\right)^{2}$. We have

$$
\|\bar{\zeta}\|_{1,1, \mathbb{S}^{*}}=\frac{1}{2}\left\|1-\left|\phi^{h, L}\right|^{2}\right\|_{0,2, \mathbb{S}^{*}}^{2}+\left\|2\left(1-\left|\phi^{h, L}\right|^{2}\right) \Re\left\{\left(\phi^{h, L}\right)^{*} \nabla_{*} \phi^{h, L}\right\}\right\|_{0,1, \mathbb{S}^{*}}
$$

By Sobolev imbedding theorems and the uniform bound on $\left\|\phi^{h, L}\right\|_{1,2, \mathbb{S}^{*}}$, we get

$$
\left|\int_{\mathbb{S}^{*}} \frac{1}{2}\left(1-\left|\phi^{h, L}\right|^{2}\right)^{2} d S^{*}-\sum_{V_{j_{1}} \in \Sigma} \frac{m\left(V_{j_{1}}\right)}{2}\left(1-\left|\phi_{j_{1}}\right|^{2}\right)^{2}\right| \leq c h .
$$

Consider a typical spherical triangle $\tilde{\tau}_{j_{1} j_{2} j_{3}}$ with vertices $\mathbf{x}_{j_{1}}, \mathbf{x}_{j_{2}}$, and $\mathbf{x}_{j_{3}}$, let

$$
a_{j_{1} j_{2}}=\frac{1}{\left\|\mathbf{x}_{j_{1}}-\mathbf{x}_{j_{2}}\right\|} \int_{\widetilde{\mathbf{x}_{j_{1}} \mathbf{x}_{j_{2}}}} \mathbf{A}_{0 s}(\mathbf{x}) d s .
$$

It is easy to see that $\overrightarrow{\mathbf{t}}_{j_{1} j_{2}}, \overrightarrow{\mathbf{t}}_{j_{2} j_{3}}, \overrightarrow{\mathbf{t}}_{j_{3} j_{1}}$ are three unit vectors for the edges of the planar triangle $\tau_{j_{1} j_{2} j_{3}}$. Then for any vector $\mathbf{v}$ (real or complex), we have [20]

$$
\begin{aligned}
m\left(\tau_{j_{1} j_{2} j_{3}}\right)|\mathbf{v}|^{2}= & \cot \theta_{j_{3}}\left\|\mathbf{x}_{j_{1}}-\mathbf{x}_{j_{2}}\right\|^{2}\left|\mathbf{v} \cdot \overrightarrow{\mathbf{t}}_{j_{1} j_{2}}\right|^{2} \\
& +\cot \theta_{j_{1}}\left\|\mathbf{x}_{j_{2}}-\mathbf{x}_{j_{3}}\right\|^{2}\left|\mathbf{v} \cdot \overrightarrow{\mathbf{t}}_{j_{2} j_{3}}\right|^{2} \\
& +\cot \theta_{j_{2}}\left\|\mathbf{x}_{j_{3}}-\mathbf{x}_{j_{1}}\right\|^{2}\left|\mathbf{v} \cdot \overrightarrow{\mathbf{t}}_{j_{3} j_{1}}\right|^{2}
\end{aligned}
$$

where the $\theta_{j}^{\prime} s$ are the corresponding angles of the triangle.

Thus, we are motivated to consider the kinetic energy:

$$
\begin{aligned}
(\mathrm{I})_{j_{1} j_{2} j_{3}}^{j_{1}, j_{2}} & :=\int_{\tau_{j_{1} j_{2} j_{3}}} \mid\left(i \nabla_{*}+\mathbf{A}_{0 *}\right) \phi^{h, L}(\mathbf{y}) \cdot \overrightarrow{\mathbf{t}}_{j_{1} j_{2}} \\
\leq & \int_{\tau_{j_{1} j_{2} j_{3}}}\left|\left(\mathbf{A}_{0 *}(\mathbf{x}) \cdot \overrightarrow{\mathbf{t}}_{j_{1} j_{2}}\right) \phi^{h, L}(\mathbf{y})-\left(a_{j_{1} j_{2}} \cdot \overrightarrow{\mathbf{t}}_{j_{1} j_{2}}\right) \phi^{h, L}\left(\mathbf{x}_{j_{2}}\right)\right|^{2} d s^{*}(\mathbf{y}) \\
\leq & 2 \int_{\tau_{j_{1} j_{2} j_{3}}}\left(\left|\mathbf{A}_{0 *}(\mathbf{y}) \cdot \overrightarrow{\mathbf{t}}_{j_{1} j_{2}}\right|^{2}\left|\phi^{h, L}(\mathbf{y})-\phi^{h, L}\left(\mathbf{x}_{j_{2}}\right)\right|^{2}\right. \\
& \left.\quad-\mid\left(\mathbf{A}_{0 *}(\mathbf{x})-\overrightarrow{\mathbf{t}}_{j_{j_{1} j_{2}}}+a_{j_{1} j_{2}} \cdot \overrightarrow{\mathbf{t}}_{j_{1} j_{2}}\right) \phi^{h, L}\left(\mathbf{x}_{j_{1} j_{2}}\right)\right)\left.\right|^{2} \mid \phi^{*}(\mathbf{y}) \\
\leq & \left.2 \| \phi^{h, L}-\left.\phi^{h, L}\left(\mathbf{x}_{j_{2}}\right)\right|^{2}\right) d s^{*}(\mathbf{y})\left\|_{0, \infty, \tau_{j_{1} j_{2} j_{3}}}^{2}\right\| \mathbf{A}_{0 *} \|_{0, \tau_{j_{1} j_{2} j_{3}}}^{2} \\
& \quad+c h^{2}\left\|\phi^{h, L}\right\|_{0, \infty, \tau_{j_{1} j_{2} j_{3}}}^{2}\left\|\mathbf{A}_{0 *}\right\|_{1, \tau_{j_{1} j_{2} j_{3}}} \cdot
\end{aligned}
$$

Here, we have used the estimate (5.6) and (5.7).

Thus, we can get

$$
\begin{aligned}
& \frac{1}{m\left(\tau_{j_{1} j_{2} j_{3}}\right)}\left(\cot \theta_{j_{3}}\left\|\mathbf{x}_{j_{1}}-\mathbf{x}_{j_{2}}\right\|^{2}(\mathrm{I})_{j_{1} j_{2} j_{3}}^{j_{1} j_{j_{2}}}\right. \\
& \left.\quad+\cot \theta_{j_{1}}\left\|\mathbf{x}_{j_{2}}-\mathbf{x}_{j_{3}}\right\|^{2}(\mathrm{I})_{j_{1} j_{2} j_{3}}^{j_{2} j_{3}}+\cot \theta_{j_{2}}\left\|\mathbf{x}_{j_{3}}-\mathbf{x}_{j_{1}}\right\|^{2}(\mathrm{I})_{j_{1} j_{2} j_{3}}^{j_{3} j_{1}}\right) \\
& \quad \leq 2 \max _{l=1,2,3}\left\|\phi^{h}-\phi^{h}\left(\mathbf{x}_{j_{l}}\right)\right\|_{0, \infty, \tau_{j_{1} j_{2} j_{3}}}^{2}\left\|\mathbf{A}_{0 *}\right\|_{0, \tau_{j_{1} j_{2} j_{3}}}^{2} \\
& \quad+\operatorname{ch}^{2}\left\|\mathbf{A}_{0 *}\right\|_{1, \tau_{j_{1} j_{2} j_{3}}}^{2}\left\|\phi^{h}\right\|_{0, \infty, \tau_{j_{1} j_{2} j_{3}}}^{2} .
\end{aligned}
$$


Since $\phi^{h, L}$ is a linear polynomial on $\tau_{j_{1} j_{2} j_{3}}$, by the inverse estimate, Holder's inequality, and the norm equivalence, we get

$$
\begin{aligned}
& \left\|\phi^{h, L}-\phi^{h, L}\left(\mathbf{x}_{j_{l}}\right)\right\|_{0, \infty, \tau_{j_{1} j_{2} j_{3}}}^{2}\left\|\mathbf{A}_{0 *}\right\|_{0, \tau_{j_{1} j_{2} j_{3}}}^{2} \\
& \quad \leq c\left\|\phi^{h, L}-\phi^{h, L}\left(\mathbf{x}_{j_{l}}\right)\right\|_{0, q, \tau_{j_{1} j_{2} j_{3}}}^{2}\left\|\mathbf{A}_{0 *}\right\|_{0, p, \tau_{j_{1} j_{2} j_{3}}}^{2},
\end{aligned}
$$

where $p>2$ and $2 / p+2 / q=1$.

By Sobolev imbedding theorems and scaling arguments again, we get

$$
\begin{aligned}
E_{1}:= & \sum_{\tau_{j_{1} j_{2} j_{3}} \in \mathcal{T}} \frac{1}{m\left(\tau_{j_{1} j_{2} j_{3}}\right)}\left\{\cot \theta_{j_{3}}\left\|\mathbf{x}_{j_{1}}-\mathbf{x}_{j_{2}}\right\|^{2}(\mathrm{I})_{j_{1} j_{2} j_{3}}^{j_{1} j_{2}}\right. \\
& \left.+\cot \theta_{j_{1}}\left\|\mathbf{x}_{j_{2}}-\mathbf{x}_{j_{3}}\right\|^{2}(\mathrm{I})_{j_{1} j_{2} j_{3}}^{j_{2} j_{3}}+\cot \theta_{j_{2}}\left\|\mathbf{x}_{j_{3}}-\mathbf{x}_{j_{1}}\right\|^{2}(\mathrm{I})_{j_{1} j_{2} j_{3}}^{j_{3} j_{1}}\right\} \\
\leq & c h^{2-4 / p}\left\|\mathbf{A}_{0 *}\right\|_{1, \mathbb{S}^{*}}^{2}\left\|\phi^{h, L}\right\|_{1, \mathbb{S}^{*}}^{2}+c h^{2}\left\|\mathbf{A}_{0 *}\right\|_{1, \mathbb{S}^{*}}^{2}\left\|\phi^{h, L}\right\|_{1, \mathbb{S}^{*}}^{2} .
\end{aligned}
$$

On the other hand, we consider

$$
\begin{aligned}
(\mathrm{II})_{j_{1} j_{2} j_{3}}^{j_{1} j_{2}} & :=\left|\left(i \nabla_{*} \phi^{h, L}\left(\mathbf{x}_{j_{2}}\right) \cdot \overrightarrow{\mathbf{t}}_{j_{1} j_{2}}+\left(a_{j_{1} j_{2}} \cdot \overrightarrow{\mathbf{t}}_{j_{1} j_{2}}\right) \phi^{h, L}\left(\mathbf{x}_{j_{2}}\right)\right)-i \beta_{j_{1} j_{2}}\right|^{2} \\
& =\left|\frac{\phi_{j_{2}}-\phi_{j_{1}}}{\left\|\mathbf{x}_{j_{1}}-\mathbf{x}_{j_{2}}\right\|}-\frac{\phi_{j_{2}} \exp \left(-i c_{j_{1} j_{2}}\right)-\phi_{j_{1}}}{\left\|\mathbf{x}_{j_{1}}-\mathbf{x}_{j_{2}}\right\|}+i \alpha_{j_{1} j_{2}} \phi^{h, L}\left(\mathbf{x}_{j_{2}}\right)\right|^{2} \\
& \leq\left|\phi^{h, L}\left(\mathbf{x}_{j_{2}}\right)\left(\frac{1-\exp \left(-i c_{j_{1} j_{2}}\right)}{\left\|\mathbf{x}_{j_{1}}-\mathbf{x}_{j_{2}}\right\|}-i \alpha_{j_{1} j_{2}}\right)\right|^{2} \\
& \leq\left|\phi^{h, L}\left(\mathbf{x}_{j_{2}}\right)\left(\frac{1-\exp \left(-i c_{j_{1} j_{2}}\right)}{\left\|\mathbf{x}_{j_{1}}-\mathbf{x}_{j_{2}}\right\|}-i \frac{c_{j_{1} j_{2}}}{\left\|\mathbf{x}_{j_{1}}-\mathbf{x}_{j_{2}}\right\|}\right)\right|^{2} \\
& \leq c\left\|\phi^{h, L}\right\|_{0, \infty, \tau_{j_{1} j_{2} j_{3}}}^{2}\left|\alpha_{j_{1} j_{2}}\right|^{4}\left\|\mathbf{x}_{j_{1}}-\mathbf{x}_{j_{2}}\right\|^{2}
\end{aligned}
$$

where $\phi_{j_{1}}=\phi^{h, L}\left(\mathbf{x}_{j_{1}}\right), \phi_{j_{2}}=\phi^{h, L}\left(\mathbf{x}_{j_{2}}\right)$ and

$$
\alpha_{j_{1} j_{2}}=a_{j_{1} j_{2}} \cdot \overrightarrow{\mathbf{t}}_{j_{1} j_{2}}=\frac{c_{j_{1} j_{2}}}{\left\|\mathbf{x}_{j_{1}}-\mathbf{x}_{j_{2}}\right\|}, \quad \beta_{j_{1} j_{2}}=\frac{\phi_{j_{2}} \exp \left(-i c_{j_{1} j_{2}}\right)-\phi_{j_{1}}}{\left\|\mathbf{x}_{j_{1}}-\mathbf{x}_{j_{2}}\right\|} .
$$

Again, by a similar argument as before, we have

$$
\begin{aligned}
E_{2}:= & \sum_{\tau_{j_{1} j_{2} j_{3} \in \mathcal{T}}}\left\{\cot \theta_{j_{3}}\left\|\mathbf{x}_{j_{1}}-\mathbf{x}_{j_{2}}\right\|^{2}(\mathrm{II})_{j_{1} j_{2} j_{3}}^{j_{1} j_{2}}\right. \\
& \left.\quad+\cot \theta_{j_{1}}\left\|\mathbf{x}_{j_{2}}-\mathbf{x}_{j_{3}}\right\|^{2}(\mathrm{II})_{j_{1} j_{2} j_{3}}^{j_{2} j_{3}}+\cot \theta_{j_{2}}\left\|\mathbf{x}_{j_{3}}-\mathbf{x}_{j_{1}}\right\|^{2}(\mathrm{II})_{j_{1} j_{2} j_{3}}^{j_{3} j_{1}}\right\} \\
\leq \quad & \operatorname{ch}^{2}\left\|\mathbf{A}_{0 *}\right\|_{1, \mathbb{S}^{2}}^{4}\left\|\phi^{h, L}\right\|_{1, \mathbb{S}^{*}}^{2} .
\end{aligned}
$$

Let

$$
\begin{aligned}
\mathcal{K}:= & \sum_{\tau_{j_{1} j_{2} j_{3} \in \mathcal{T}}}\left\{\cot \theta_{j_{3}}\left\|\mathbf{x}_{j_{1}}-\mathbf{x}_{j_{2}}\right\|^{2}\left|\beta_{j_{1} j_{2}}\right|^{2}\right. \\
& \left.\quad+\cot \theta_{j_{2}}\left\|\mathbf{x}_{j_{2}}-\mathbf{x}_{j_{3}}\right\|^{2}\left|\beta_{j_{2} j_{3}}\right|^{2}+\cot \theta_{j_{1}}\left\|\mathbf{x}_{j_{3}}-\mathbf{x}_{j_{1}}\right\|^{2}\left|\beta_{j_{3} j_{1}}\right|^{2}\right\} .
\end{aligned}
$$

Then, by the estimate on $\phi^{h}$, we get the error estimate

$$
\begin{aligned}
& \left.\left|\int_{\mathbb{S}^{*}}\right|\left(i \nabla_{*}+\mathbf{A}_{0 *}\right) \phi^{h, L}\right|^{2} d S^{*}-\mathcal{K} \mid \leq c\left(E_{1}+E_{2}\right) \\
& \quad \leq c h^{2-4 / p}\left\|\mathbf{A}_{0 *}\right\|_{1, \mathbb{S}^{*}}^{2}\left(1+h^{4 / p}+h^{4 / p}\left\|\mathbf{A}_{0 *}\right\|_{1, \mathbb{S}^{*}}^{2}\right)\left\|\phi^{h}\right\|_{1, \mathbb{S}^{*}}^{2}
\end{aligned}
$$

for any $p \geq 2$ and $h$ small. 
Using mesh regularity and approximation properties of planar triangles $\tau_{j_{1} j_{2} j_{3}}$ to spherical triangles $\tilde{\tau}_{j_{1} j_{2} j_{3}}$, it is easy to find that for any two triangles $\tau_{j_{i_{1}} j_{2} j_{3}}$ and $\tau_{j_{i_{2}} j_{2} j_{3}}$ sharing the common edge $\mathbf{x}_{j_{2}} \mathbf{x}_{j_{3}}$, we have

$$
\left|\cot \theta_{j_{i_{1}}}+\cot \theta_{j_{i_{2}}}-\frac{m\left(\Gamma_{j_{2} j_{3}}\right)}{\left\|\mathbf{x}_{j_{2}}-\mathbf{x}_{j_{3}}\right\|}\right| \leq c h^{2}\left(\cot \theta_{j_{i_{1}}}+\cot \theta_{j_{i_{2}}}\right) \text {. }
$$

Let

$$
\begin{aligned}
\tilde{\mathcal{K}} & :=\frac{1}{2} \sum_{j=1}^{n} \sum_{k \in \chi_{j}} \frac{m\left(\Gamma_{j k}\right)}{\left\|\mathbf{x}_{j}-\mathbf{x}_{k}\right\|}\left|\psi_{k} \exp \left(-i c_{j k}\right)-\psi_{j}\right|^{2} \\
& =\frac{1}{2} \sum_{j=1}^{n} \sum_{k \in \chi_{j}} \frac{m\left(\Gamma_{j k}\right)}{\left\|\mathbf{x}_{j}-\mathbf{x}_{k}\right\|}\left\|\mathbf{x}_{j}-\mathbf{x}_{k}\right\|^{2}\left|\beta_{j k}\right|^{2} .
\end{aligned}
$$

Then $|\tilde{\mathcal{K}}-\mathcal{K}| \leq c h^{2} \mathcal{K}$, and consequently,

$$
\begin{aligned}
|\tilde{\mathcal{K}}-\mathcal{K}| \leq & c h^{2}\left(\left.\left|\mathcal{K}-\int_{\mathbb{S}^{*}}\right|\left(i \nabla_{*}+\mathbf{A}_{0 *}\right) \phi^{h, L}\right|^{2} d S^{*} \mid\right. \\
& \left.\quad+\int_{\mathbb{S}^{*}}\left|\left(i \nabla_{*}+\mathbf{A}_{0 *}\right) \phi^{h, L}\right|^{2} d S^{*}\right) \\
\leq & c h^{2}\left(\left\|\phi^{h, L}\right\|_{1, \mathbb{S}^{*}}+\left\|\mathbf{A}_{0 *}\right\|_{1, \mathbb{S}^{*}}^{2}\left\|\phi^{h, L}\right\|_{1, \mathbb{S}^{*}}^{2}\right) .
\end{aligned}
$$

Thus, we have

$$
\begin{aligned}
& \left.\left|\int_{\mathbb{S}^{*}}\right|\left(i \nabla_{*}+\mathbf{A}_{0 *}\right) \phi^{h, L}\right|^{2} d S^{*}-\tilde{\mathcal{K}} \mid \\
& \quad \leq c h^{2-4 / p}\left(1+\left\|\mathbf{A}_{0 *}\right\|_{1, \mathbb{S}^{*}}^{2}\right)\left(1+h^{4 / p}+h^{4 / p}\left\|\mathbf{A}_{0 *}\right\|_{1, \mathbb{S}^{*}}^{2}\right)\left\|\phi^{h}\right\|_{1, \mathbb{S}^{*}}^{2} .
\end{aligned}
$$

Taking $p$ sufficiently large in (5.14) with (5.13), we obtain

$$
\left|\mathcal{F}^{L}\left(\phi^{h, L}\right)-\mathcal{F}^{h}\left(\vec{\phi}^{h}\right)\right| \leq c h,
$$

which directly induces (5.10).

Now let $\psi_{*}$ be a global minimizer of problem (2.2); i.e.,

$$
\mathcal{F}\left(\psi_{*}\right)=\min _{\mathcal{H}^{1}\left(\mathbb{S}^{2}\right)} \mathcal{F}
$$

Let $I^{h} \psi_{*}$ be an approximate piecewise linear approximation of $\psi_{*}$ (the standard elliptic projection or an interpolant using the averages of $\psi_{*}$ over the Voronoi regions $\left\{V_{j}\right\}$ as values at the vertices $\left\{x_{j}\right\}$ ).

It is straightforward to see that the bounds on the energy functional imply

$$
\left\|I^{h} \psi_{*}\right\|_{1, \mathbb{S}^{2}} \leq c\left\|\psi_{*}\right\|_{1, \mathbb{S}^{2}} .
$$

It follows that

Lemma 5.5. Using the definitions given above, we have

$$
\mathcal{F}\left(I^{h} \psi_{*}\right)=\mathcal{F}\left(\psi_{*}\right)+o(1) \quad \text { as } h \rightarrow 0
$$

and

$$
\mathcal{F}\left(I^{h} \psi_{*}\right)=\mathcal{F}^{h}\left(\vec{\psi}_{*}^{h}\right)+o(1) \quad \text { as } \quad h \rightarrow 0,
$$

where $\vec{\psi}_{*}^{h}=\left(I^{h} \psi_{*}\left(\mathbf{x}_{1}\right), I^{h} \psi_{*}\left(\mathbf{x}_{2}\right), \ldots, I^{h} \psi_{*}\left(\mathbf{x}_{n}\right)\right)$. 
For any $h$, let $\vec{\psi}^{h}$ be a minimizer of $\mathcal{F}^{h}$; i.e.,

$$
\mathcal{F}^{h}\left(\vec{\psi}^{h}\right)=\min _{R^{\wedge \mathrm{N}}} \mathcal{F}^{h}
$$

It is easy to see that $\mathcal{F}^{h}\left(\vec{\psi}^{h}\right)$ is uniformly bounded.

Let $\psi^{h}$ be the approximate piecewise linear approximation of the data $\vec{\psi}^{h}$ in $\mathbb{S}^{2}$. Then

$$
\left\|\psi^{h}\right\|_{1, \mathbb{S}^{2}} \leq c
$$

for some generic constant $c>0$.

We now prove the convergence theorem.

Theorem 5.6. For any $h$, let $\vec{\psi}^{h}$ and $\psi^{h}$ be defined as above. Then as $h \rightarrow 0$, there is a subsequence of $\left\{\psi^{h}\right\}$ that converges to a global minimizer of $\mathcal{F}$ in $\mathcal{H}^{1}(\Omega)$, and

$$
\lim _{h \rightarrow 0} \mathcal{F}^{h}\left(\vec{\psi}^{h}\right)=\min _{\mathcal{H}^{1}\left(\mathbb{S}^{2}\right)} \mathcal{F}
$$

Proof. Let $\psi_{*}$ be a global minimizer of $\mathcal{F}$. By definition,

$$
\mathcal{F}\left(\psi_{*}\right) \leq \mathcal{F}\left(\psi^{h}\right) .
$$

For any $\delta>0$, if $h$ small enough, we have, by Lemma 5.4,

$$
\mathcal{F}\left(\psi^{h}\right) \leq \mathcal{F}^{h}\left(\vec{\psi}^{h}\right)+\delta .
$$

Thus,

$$
\mathcal{F}\left(\psi_{*}\right) \leq \mathcal{F}^{h}\left(\vec{\psi}_{*}^{h}\right)+\delta
$$

It follows from earlier estimates in Lemma 5.5 that, for small $h$,

$$
\mathcal{F}^{h}\left(\vec{\psi}_{*}^{h}\right) \leq \mathcal{F}\left(\psi_{*}\right)+\delta .
$$

So,

$$
\mathcal{F}\left(\psi_{*}\right) \leq \mathcal{F}\left(\psi^{h}\right) \leq \mathcal{F}\left(\psi_{*}\right)+2 \delta
$$

and

$$
\mathcal{F}\left(\psi_{*}\right) \leq \mathcal{F}^{h}\left(\vec{\psi}^{h}\right)+\delta \leq \mathcal{F}\left(\psi_{*}\right)+2 \delta
$$

If we let $\delta \rightarrow 0$, we see that $\left\{\psi^{h}\right\}$ forms a minimizing sequence of $\mathcal{F}$; thus, it has a weakly convergent subsequence $\left\{\psi_{n}^{h}\right\}$ that converges to a global minimizer of $\mathcal{F}$. Using results in functional analysis, we see that the subsequence also converges strongly in $\mathcal{H}^{1}\left(\mathbb{S}^{2}\right)$. Moreover, we consequently have

$$
\lim _{h \rightarrow 0} \mathcal{F}^{h}\left(\vec{\psi}^{h}\right)=\lim _{h \rightarrow 0} \mathcal{F}\left(\psi^{h}\right)=\min _{\mathcal{H}^{1}\left(\mathbb{S}^{2}\right)} \mathcal{F} .
$$

The above theorem gives the convergence of the discrete approximation under minimal regularity assumptions on the exact solution. If smoothness assumptions on the exact solutions can be made, we have the following results, which indicate the superiority of the SCVT grid.

Corollary 5.7. Given $\psi \in \mathcal{H}^{2}(\Omega)$, let $\vec{\psi}^{h}$ and $\psi^{h}$ be defined as above for a SCVT grid. Then, for any $\delta>0$,

$$
\mathcal{F}^{h}\left(\vec{\psi}^{h}\right)=\mathcal{F}(\psi)+O\left(h^{2-\delta}\right) \quad \text { as } \quad h \rightarrow 0 .
$$


Proof. With the smoothness assumption on $\psi$, we can utilize the property of the SCVT to get that

$$
\left|\int_{\mathbb{S}^{2}} \zeta d S-\sum_{V_{j_{1}} \in \Sigma} \zeta\left(\mathbf{x}_{j_{1}}\right) m\left(V_{j_{1}}\right)\right| \leq c h^{2}\|\zeta\|_{2, \mathbb{S}^{2}},
$$

where $\zeta=\frac{1}{2}\left(1-|\psi|^{2}\right)^{2}$ is in $\mathcal{H}^{2}\left(\mathbb{S}^{2}\right)$, and $c$ is a generic constant independent of $h$.

Note that a scaling argument leads to

$$
\left\|\nabla_{s} \psi-\nabla_{s} I^{h} \psi\right\|_{0, \tilde{\tau}_{j_{1} j_{2} j_{3}}} \leq \operatorname{ch}\|\psi\|_{2, \tilde{\tau}_{j_{1} j_{2} j_{3}}}, \quad j=j_{1}, j_{2}, j_{3},
$$

for some constant $c>0$. Then, following the proof of the earlier theorems, we can get that

$$
\left|\mathcal{F}^{h}\left(\vec{\psi}^{h}\right)-\mathcal{F}(\psi)\right| \leq c h^{2-1 / p} \quad \text { as } \quad h \rightarrow 0
$$

for any $p>2$, which can be arbitrarily large.

We note that if the grid used is not a SCVT grid, only first order estimates of the type

$$
\mathcal{F}^{h}\left(\vec{\psi}^{h}\right)=\mathcal{F}(\psi)+O(h) \quad \text { as } \quad h \rightarrow 0
$$

may be expected since (5.16) may not be always valid for arbitrary spherical Voronoi tessellations. Thus, the SCVT allows an almost full extra order of accuracy over the arbitrary Voronoi-Delaunay grids on the sphere. It is also possible to derive error estimates for the finite volume approximations using arguments along the lines of [17]. We leave this to future works.

\section{Gauge invariant gradient Recovery and Field COMPutation}

Once the discrete solution $\left\{\psi_{j}\right\}$ is computed, we can readily obtain the scalar field $\left\{\left|\psi_{j}\right|^{2}\right\}$ which described the local state: the positions $\left\{j|| \psi_{j} \mid=0\right\}$ are called the vortices as the superconducting carrier density $\left(\left|\psi_{j}\right|^{2}\right)$ is zero; on the other hand, the points $\left\{j|| \psi_{j} \mid \approx 1\right\}$ represent superconducting region.

Another interesting physical quantity is the supercurrent

$$
\mathbf{J}_{s}=\Im\left\{\psi^{*} \nabla_{s} \psi\right\}-\mathbf{A}_{0 s}|\psi|^{2}=-\Re\left\{\psi^{*}\left(i \nabla_{s} \psi+\mathbf{A}_{0 s} \psi\right)\right\}, \quad \text { on } S^{2} .
$$

Since it involves the gradient computation, an accurate scheme is required. One way is to use the superconvergent gradient recovery technique proposed in [17] for the SCVTs. That is to do the two terms $\psi^{*} \nabla_{s} \psi$ and $\mathbf{A}_{0 s}|\psi|^{2}$ separately. After all, only the gradient term $\nabla_{s} \psi$ needs better recovery scheme.

However, in order to preserve the gauge invariance, we here propose another approach for superconvergent gradient recovery which gives an accurate computation of $i \nabla_{s} \psi+\mathbf{A}_{0 s} \psi$ and the supercurrent $\mathbf{J}_{s}$ while still preserves discrete gauge invariance.

For any generator $\mathbf{x}_{j}$, let us define an element patch $V_{\mathbf{x}_{j}}=\bigcup_{\mathbf{x}_{j} \in \tau_{j j_{1} j_{2}}} \tau_{j j_{1} j_{2}}$; that is, the union of all spherical triangles in the dual spherical Delaunay triangulation that contain $\mathbf{x}_{j}$ as one of its vertices. We first project $V_{\mathbf{x}_{j}}$ onto the tangential plane $S_{\mathbf{x}_{j}}$ of $\mathbb{S}^{2}$ at $\mathbf{x}_{j}\left(S_{\mathbf{x}_{j}}\right.$ is perpendicular to $\overrightarrow{\mathbf{n}}_{\mathbf{x}_{j}}$ at $\left.\mathbf{x}_{j}\right)$. Let $\vec{e}_{1, \mathbf{x}_{j}}, \vec{e}_{2, \mathbf{x}_{j}}$ be any given orthonormal basis of $S_{\mathbf{x}_{j}}$. We now move $S_{\mathbf{x}_{j}}$ to the $(x, y)$-plane via an affine map satisfying that $\overrightarrow{\mathbf{n}}_{\mathbf{x}_{j}}$ is mapped to the $z$-axis and $\vec{e}_{1, \mathbf{x}_{j}}$ to the $x$-axis and $\vec{e}_{2, \mathbf{x}_{j}}$ to the $y$-axis (see Figure 2). We then define a map $H_{\mathbf{x}_{j}}: V_{\mathbf{x}_{j}} \rightarrow \mathbb{R}^{2}$ by the above procedure such that $V_{\mathbf{x}_{j}}^{\prime}=H_{\mathbf{x}_{j}}\left(V_{\mathbf{x}_{j}}\right)$. 


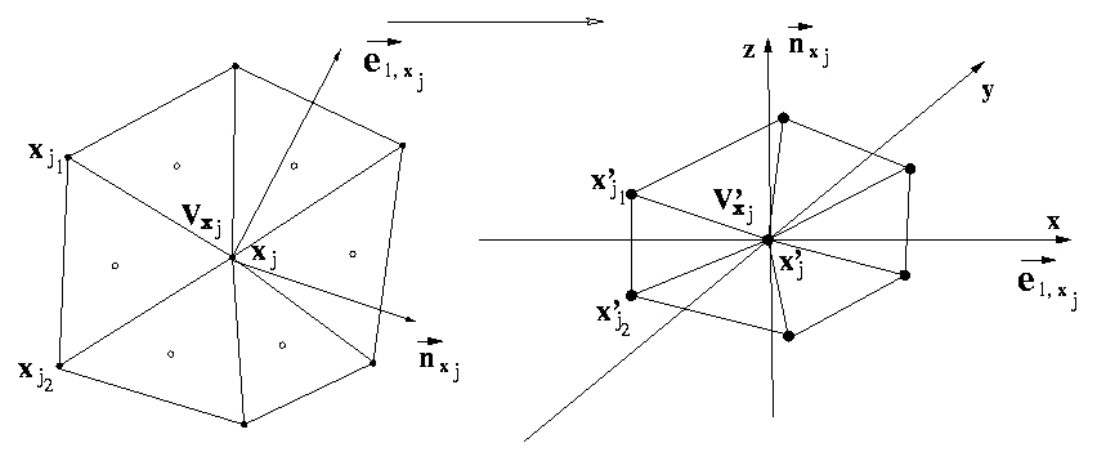

FIGURE 2. The mapping $H_{\mathbf{x}_{j}}$.

On each planar triangle $\triangle \mathbf{x}_{j}^{\prime} \mathbf{x}_{j_{1}}^{\prime} \mathbf{x}_{j_{2}}^{\prime}=H_{\mathbf{x}_{j}}\left(\tau_{j j_{1} j_{2}}\right)$, we uniquely determine a linear function $\bar{\psi}_{j j_{1} j_{2}}$ by setting

$$
\bar{\psi}_{j j_{1} j_{2}}\left(\mathbf{x}_{l}^{\prime}\right)=\psi^{h}\left(\mathbf{x}_{l}\right)=\psi_{l}, \quad l=j, j_{1}, j_{2}
$$

Then, we compute the gauge invariant approximations to the components of $i \nabla_{s} \psi+$ $\mathbf{A}_{0 s} \psi$ along the $\mathbf{x}_{j}^{\prime} \mathbf{x}_{j_{1}}^{\prime}$ and $\mathbf{x}_{j}^{\prime} \mathbf{x}_{j_{2}}^{\prime}$ directions, respectively:

$$
v_{j j_{k}}=i \frac{\psi_{j_{k}} \exp \left(-i c_{j j_{k}}\right)-\psi_{j}}{\left\|\mathbf{x}_{j}-\mathbf{x}_{j_{k}}\right\|}, \quad k=1,2
$$

Clearly, from the vector decomposition and the fact that $\bar{\psi}_{j j_{1} j_{2}}$ is linear on $\triangle \mathbf{x}_{j}^{\prime} \mathbf{x}_{j_{1}}^{\prime} \mathbf{x}_{j_{2}}^{\prime}$, we know that there exists a unique set $\left\{s_{k l}\right\}_{k, l=1}^{2}$ such that

$$
\nabla_{\mathbf{x}^{\prime}} \psi_{j j_{1} j_{2}}=\left(\begin{array}{cc}
s_{11} & s_{12} \\
s_{21} & s_{22}
\end{array}\right)\left(\begin{array}{c}
\nabla \bar{\psi}_{j j_{1} j_{2}} \cdot \overrightarrow{\mathbf{t}}_{\mathbf{x}_{j}^{\prime} \mathbf{x}_{j_{1}}^{\prime}} \\
\nabla \bar{\psi}_{j j_{1} j_{2}} \cdot \overrightarrow{\mathbf{t}}_{\mathbf{x}_{j}^{\prime} \mathbf{x}_{j_{2}}^{\prime}}
\end{array}\right),
$$

where $\overrightarrow{\mathbf{t}}_{\mathbf{x}_{j}^{\prime} \mathbf{x}_{l}^{\prime}}$ denotes the unit vector from $\mathbf{x}_{j}^{\prime}$ to $\mathbf{x}_{l}^{\prime}$ for $l=j_{1}, j_{2}$. Since $\mathbf{x}_{j}^{\prime}$ is the origin, if we write $\mathbf{x}_{j_{1}}^{\prime}=\left(x_{1}, y_{1}\right)$ and $\mathbf{x}_{j_{2}}^{\prime}=\left(x_{2}, y_{2}\right)$, then in fact we can get

$$
s_{11}=\frac{y_{2} r_{1}}{Q}, \quad s_{12}=-\frac{y_{1} r_{2}}{Q}, \quad s_{21}=-\frac{x_{2} r_{1}}{Q}, \quad s_{12}=\frac{x_{1} r_{2}}{Q}
$$

where

$$
r_{1}=\sqrt{x_{1}^{2}+y_{1}^{2}}, \quad r_{2}=\sqrt{x_{2}^{2}+y_{2}^{2}}, \quad Q=x_{1} y_{2}-x_{2} y_{1} .
$$


Now we set

$$
\left(\begin{array}{l}
D_{j j_{1} j_{2}}^{1} \\
D_{j j_{1} j_{2}}^{2}
\end{array}\right)=\left(\begin{array}{ll}
s_{11} & s_{12} \\
s_{21} & s_{22}
\end{array}\right)\left(\begin{array}{c}
v_{j j_{1}} \\
v_{j j_{2}}
\end{array}\right)
$$

Finally, we approximate $i \nabla_{s} \psi+\mathbf{A}_{0 s} \psi$ at $\mathbf{x}_{j}$ by

$$
\left(i \nabla_{s} \psi+\mathbf{A}_{0 s} \psi\right)\left(\mathbf{x}_{j}\right) \approx \alpha_{j}=\frac{1}{q} \sum_{\tau_{j j_{1} j_{2}} \subset V_{\mathbf{x}_{j}}}\left(D_{j j_{1} j_{2}}^{1} \vec{e}_{1, \mathbf{x}_{j}}+D_{j j_{1} j_{2}}^{2} \vec{e}_{2, \mathbf{x}_{j}}\right)
$$

where $q=\operatorname{Card}\left(\left\{\tau_{j j_{1} j_{2}} \mid \tau_{j j_{1} j_{2}} \subset V_{\mathbf{x}_{j}}\right\}\right)$. Then we compute the discrete supercurrent $\mathbf{J}_{s}$ at $\mathbf{x}_{j}$ by

$$
\mathbf{J}_{s}\left(\mathbf{x}_{j}\right) \approx-\Re\left\{\psi\left(\mathbf{x}_{j}\right) \alpha_{j}\right\}
$$

Next, we consider the computation of the induced magnetic field. Although for very thin shells, the applied magnetic field, to the leading order, penetrates through the sample completely $\left(\operatorname{curl} \mathbf{A}_{0}=H \vec{z}\right.$ ), it is also of interest to study the higher order corrections, in particular, the next order term $\mathbf{H}_{1}$ given by the solution of the following three-dimensional Maxwell equations [5]:

$$
\operatorname{div} \mathbf{H}_{1}=0, \quad \operatorname{curl} \mathbf{H}_{1}=0, \quad \text { in } \mathbb{R}^{3}
$$

with interface condition

$$
\left[\mathbf{H}_{1} \times \mathbf{n}\right]_{s}=\mathbf{J}_{s} \times \mathbf{n} \quad \text { on } \mathbb{S}^{2}
$$

where $\mathbf{n}$ is the unit outer normal to the sphere and $[\cdot]_{s}$ denotes the jump across the spherical surface (the difference between the exterior value and the interior value), and $\mathbf{H}_{1} \rightarrow 0$ at infinity.

The solution $\mathbf{H}_{1}$ can be computed via an integral representation: for any $\mathbf{y} \in \mathbb{R}^{3}$,

$$
\mathbf{H}_{1}(\mathbf{y})=\int_{\mathbb{S}^{2}} \nabla \frac{1}{\|\mathbf{y}-\mathbf{x}\|} \times \mathbf{J}_{s}(\mathbf{x}) d S_{\mathbf{x}}
$$

The total corrected field is given by $\mathbf{H}=H \vec{z}+\delta \mathbf{H}_{1}$, where $\delta$ represents the thickness of the thin spherical shell.

It is easy to see that

$$
\int_{S^{2}} \frac{1}{\|\mathbf{y}-\mathbf{x}\|} d S_{\mathbf{x}}= \begin{cases}4 \pi r, & \|\mathbf{y}\| \leq r \\ 4 \pi r^{2} /\|\mathbf{y}\|, & \|\mathbf{y}\|>r\end{cases}
$$

So for any $\mathbf{y} \in \mathbb{R}^{3}-\mathbb{S}^{2}$, we get that

$$
\mathbf{G}(\mathbf{y})=\int_{\mathbb{S}^{2}} \nabla \frac{1}{\|\mathbf{y}-\mathbf{x}\|} d S_{\mathbf{x}}= \begin{cases}0, & \|\mathbf{y}\|<r \\ -4 \pi r^{2} \mathbf{y} /\|\mathbf{y}\|^{3}, & \|\mathbf{y}\|>r\end{cases}
$$


and for any $\mathbf{y} \in \mathbb{S}^{2}$,

$$
\left\{\begin{array}{l}
\mathbf{G}^{-}(\mathbf{y})=\lim _{\mathbf{y}^{\prime} \rightarrow \mathbf{y},\left\|\mathbf{y}^{\prime}\right\|<r} \int_{\mathbb{S}^{2}} \nabla \frac{1}{\left\|\mathbf{y}^{\prime}-\mathbf{x}\right\|} d S_{\mathbf{x}}=0, \\
\mathbf{G}^{+}(\mathbf{y})=\lim _{\mathbf{y}^{\prime} \rightarrow \mathbf{y},\left\|\mathbf{y}^{\prime}\right\|>r} \int_{\mathbb{S}^{2}} \nabla \frac{1}{\left\|\mathbf{y}^{\prime}-\mathbf{x}\right\|} d S_{\mathbf{x}}=-4 \pi \mathbf{y} /\|\mathbf{y}\|,
\end{array}\right.
$$

which leads to the jump discontinuity of the applied magnetic field across the spherical surface.

To accurately compute the $\mathbf{H}_{1}$, we need to resolve the numerical singularity when $\mathbf{y}$ approaches $\mathbb{S}^{2}$. Let us choose $\mathbf{x}_{j_{\mathbf{y}}} \in\left\{\mathbf{x}_{j}\right\}_{j=1}^{n}$ such that

$$
\left\|\mathbf{x}_{j_{\mathbf{y}}}-\mathbf{y}\right\|<\|\mathbf{x}-\mathbf{y}\| \text { for all } \mathbf{x} \in\left\{\mathbf{x}_{j}\right\}_{j=1}^{n}-\mathbf{x}_{j_{\mathbf{y}}} .
$$

For any $\mathbf{y} \in \mathbb{R}^{3}-\mathbb{S}^{2}$, we compute

$$
\begin{aligned}
\mathbf{H}_{1}(\mathbf{y}) \approx \overline{\mathbf{H}}_{1}(\mathbf{y}) & =\mathbf{G}(\mathbf{y}) \times \mathbf{J}_{s}\left(\mathbf{x}_{j_{\mathbf{y}}}\right) \\
& +\sum_{j \neq j_{\mathbf{y}}} m\left(V_{j}\right)\left(\nabla\left(\|\mathbf{y}-\mathbf{x}\|^{-1}\right)\left(\mathbf{x}_{j}\right) \times\left(\mathbf{J}_{s}\left(\mathbf{x}_{j}\right)-\mathbf{J}_{s}\left(\mathbf{x}_{j_{\mathbf{y}}}\right)\right)\right) .
\end{aligned}
$$

If $\mathbf{y} \in \mathbb{S}^{2}$, then replace $\mathbf{G}$ by $\mathbf{G}^{-}$and $\mathbf{G}^{+}$, respectively, in (6.9) to get the interior limit and the exterior limit of $\mathbf{H}_{1}(\mathbf{y})$ across the sphere surface.

Let us estimate the error of the approximation (6.9):

$$
\begin{aligned}
\mathbf{H}_{1}(\mathbf{y})-\overline{\mathbf{H}}_{1}(\mathbf{y})= & \int_{\mathbb{S}^{2}} \nabla\|\mathbf{y}-\mathbf{x}\|^{-1} \times\left(\mathbf{J}_{s}(\mathbf{x})-\mathbf{J}_{s}\left(\mathbf{x}_{j_{\mathbf{y}}}\right)\right) d S_{\mathbf{x}} \\
& -\sum_{j \neq j_{\mathbf{y}}} m\left(V_{j}\right)\left(\nabla\left(\|\mathbf{y}-\mathbf{x}\|^{-1}\right)\left(\mathbf{x}_{j}\right) \times\left(\mathbf{J}_{s}\left(\mathbf{x}_{j}\right)-\mathbf{J}_{s}\left(\mathbf{x}_{j_{\mathbf{y}}}\right)\right)\right) \\
= & \sum_{j \neq j_{\mathbf{y}}}\left(\int_{V_{j}} \nabla\|\mathbf{y}-\mathbf{x}\|^{-1} \times\left(\mathbf{J}_{s}(\mathbf{x})-\mathbf{J}_{s}\left(\mathbf{x}_{j_{\mathbf{y}}}\right)\right) d S_{\mathbf{x}}\right. \\
& \left.-m\left(V_{j}\right)\left(\nabla\left(\|\mathbf{y}-\mathbf{x}\|^{-1}\right)\left(\mathbf{x}_{j}\right) \times\left(\mathbf{J}_{s}\left(\mathbf{x}_{j}\right)-\mathbf{J}_{s}\left(\mathbf{x}_{j_{\mathbf{y}}}\right)\right)\right)\right) \\
& +\int_{V_{j_{\mathbf{y}}}} \nabla\|\mathbf{y}-\mathbf{x}\|^{-1} \times\left(\mathbf{J}_{s}(\mathbf{x})-\mathbf{J}_{s}\left(\mathbf{x}_{j_{\mathbf{y}}}\right)\right) d S_{\mathbf{x}} \\
= & I_{1}+I_{2}
\end{aligned}
$$

Suppose that $\mathbf{J}_{s} \in\left(C^{2}\left(\mathbb{S}^{2}\right)\right)^{3}$. It is easy to see that there exists a constant $C>0$ such that for any $\mathbf{x} \in \mathbb{S}^{2}$,

$$
\left|\nabla\|\mathbf{y}-\mathbf{x}\|^{-1} \times\left(\mathbf{J}_{s}(\mathbf{x})-\mathbf{J}_{s}\left(\mathbf{x}_{j_{\mathbf{y}}}\right)\right)\right|<C .
$$

We get

$$
\left|I_{2}\right| \leq C m\left(V_{j_{\mathbf{y}}}\right) \leq c h^{2} .
$$

On the other hand, using the special properties of SCVT [17], by the CauchySchwartz inequality, we know that

$$
\left|I_{1}\right| \leq \sum_{j \neq j_{\mathbf{y}}} c h^{2}\left(m\left(V_{j}\right)\right)^{1 / 2}\left\|\mathbf{J}_{s}\right\|_{2, V_{j}} \leq c h^{2}\left\|\mathbf{J}_{s}\right\|_{2, \mathbb{S}^{2}} .
$$

By (6.10) and (6.11), we see that (6.9) gives a high-quality quadrature rule on the sphere for accurately computing the correction to the magnetic field. 

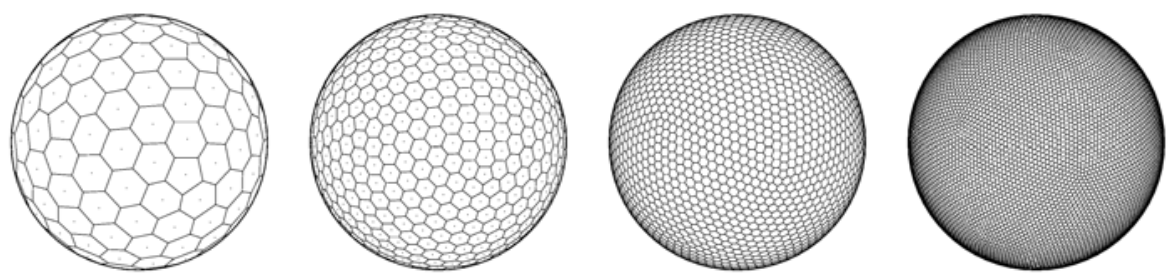

Figure 3. SCVT with a constant density for $n=162,642,2562,10242$.

\section{NumERICAL EXPERIMENTS}

To solve the finite volume equations for the nonlinear G-L model, the so-called Nonlinear Conjugate Gradient method [27] is used. Other nonlinear minimization methods can also be applied, such as Newton's method or other descent methods. In the simulation reported here, we take the unit sphere $(r=1)$ and set $\epsilon=0.2$. The spherical centroidal Voronoi meshes with $n=162,642,2562,10242$ are used; see Figure 3 ,

TABLE 1. Computational results for $H=15.0$.

\begin{tabular}{|c||c|c|c|c|}
\hline$n$ & 162 & 642 & 2562 & 10242 \\
\hline $\mathcal{F}^{h}$ & 68.7961 & 73.1503 & 74.1873 & 74.4508 \\
\hline$\left|\mathcal{F}^{h}-\mathcal{F}^{*}\right| /\left|\mathcal{F}^{*}\right|$ & 0.0761 & 0.0175 & 0.0036 & - \\
\hline$\left\|\psi^{h}-\psi^{*}\right\|_{0, \mathbb{S}^{2}} /\left\|\psi^{*}\right\|_{0, \mathbb{S}^{2}}$ & 0.2669 & 0.0662 & 0.0186 & - \\
\hline
\end{tabular}
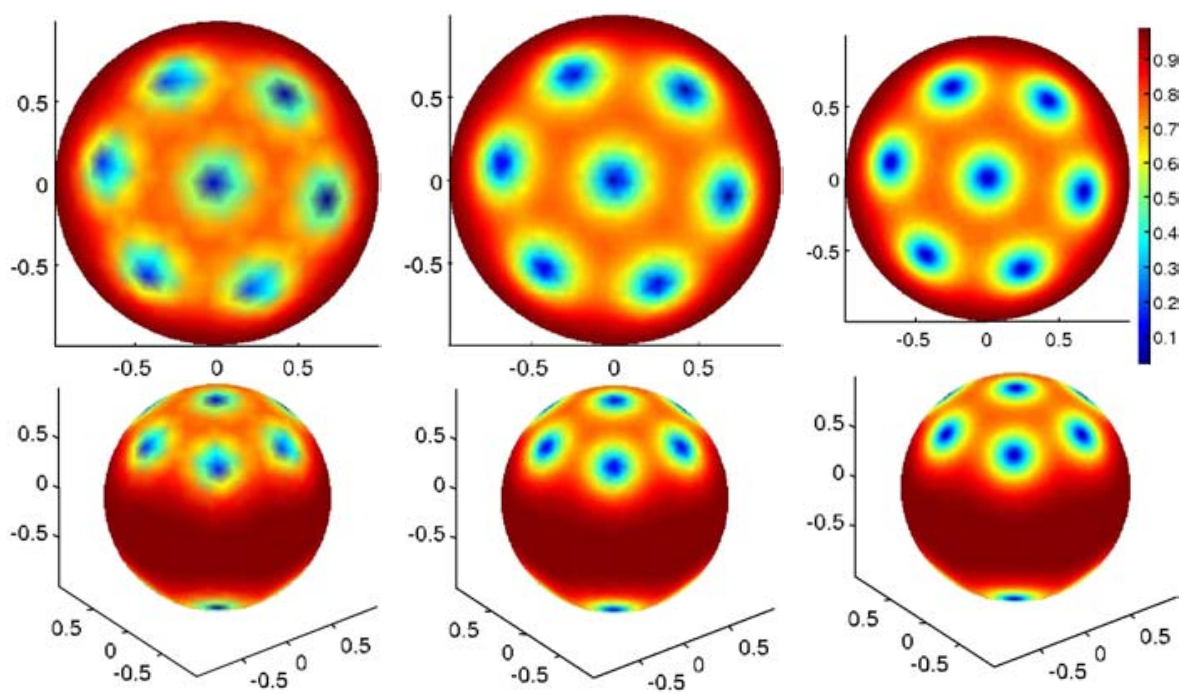

Figure 4. The solution $\left|\psi^{h}\right|$ for $H=15.0$. Top: top view; bottom: 3 -D view; left: $n=642$; middle: $n=2562$; right: $n=10242$. 
When the applied magnetic field is absent $(H=0)$, the global energy minimizer is given by a pure superconducting solution $\psi=\exp (i \theta)$ for some constant angle $\theta$. In our simulation, with the initial iteration given by $\psi(\mathbf{x})=0.5 \exp \left(i \frac{\pi}{4}\right)$ everywhere, a pure superconducting solution $\psi=\exp \left(i \frac{\pi}{4}\right)$ is found numerically; see the first picture of Figure 8 . For $H$ large enough, we expect to see vortex nucleation; that is, $\psi(\mathbf{x})=0$ at some points $\mathbf{x} \in \mathbb{S}^{2}$, and due to the symmetry of the sphere, vortices appear in pairs (with vortex and antivortex on two hemispheres). For larger and larger $H$, more and more vortices nucleate (again in pairs). A pathfollowing approach is adopted here to probe the energy landscape by varying the parameter $H$.

To find solutions with vortices, due to the energy barrier [3, 4, sometimes we intensionally plant some vortex-like structures. For instance, an initial guess of the
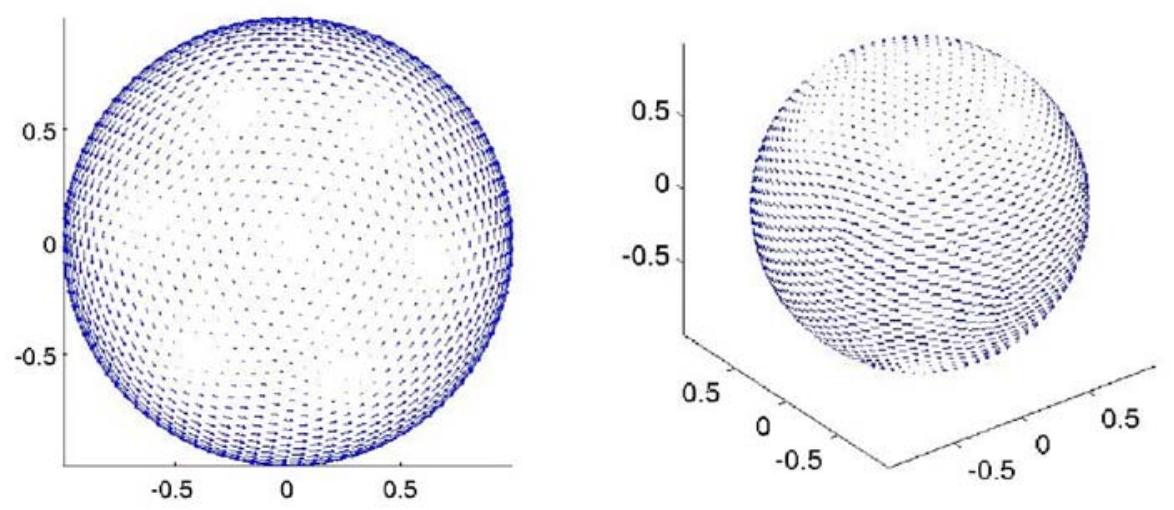

FiguRE 5. Supercurrent $\mathbf{J}_{s}$. Left: top view; right: 3-D view.
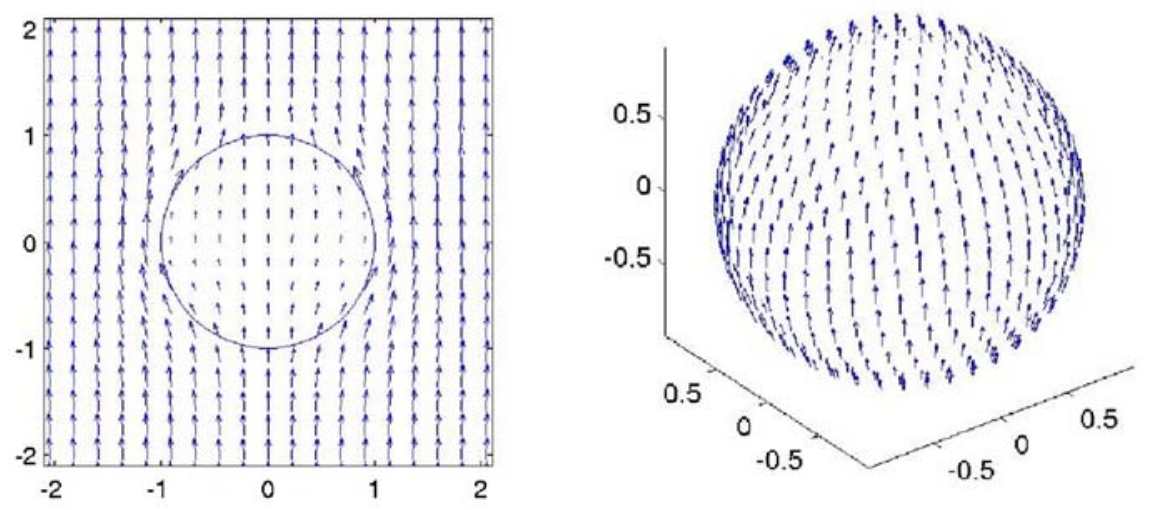

Figure 6 . Corrected magnetic field $\mathbf{H}$ with $\delta=0.1$. Left: on the plane $y=0$; right: on the surface $\{\mathbf{x} \mid\|\mathbf{x}\|=1.1\}$. 
TABLE 2. Minimum energy $\mathcal{F}^{h}$ vs. magnetics field strength $H$.

\begin{tabular}{|c||c|c|c|c|c|c|c|}
\hline$H$ & 0.0 & 1.0 & 2.0 & 3.0 & 4.0 & 5.0 & 6.0 \\
\hline $\mathcal{F}^{h}$ & 0.0000 & 2.0863 & 8.2457 & 17.2853 & 19.9073 & 26.3519 & 32.2881 \\
\hline \hline$H$ & 7.0 & 8.0 & 9.0 & 10.0 & 11.0 & 12.0 & 13.0 \\
\hline $\mathcal{F}^{h}$ & 37.0270 & 41.7372 & 45.4901 & 50.6589 & 53.6079 & 59.3215 & 61.6361 \\
\hline \hline$H$ & 14.0 & 15.0 & 16.0 & 17.0 & 18.0 & 19.0 & 20.0 \\
\hline $\mathcal{F}^{h}$ & 66.4742 & 68.5107 & 72.8404 & 73.5258 & 78.6327 & 81.0200 & 84.2344 \\
\hline
\end{tabular}

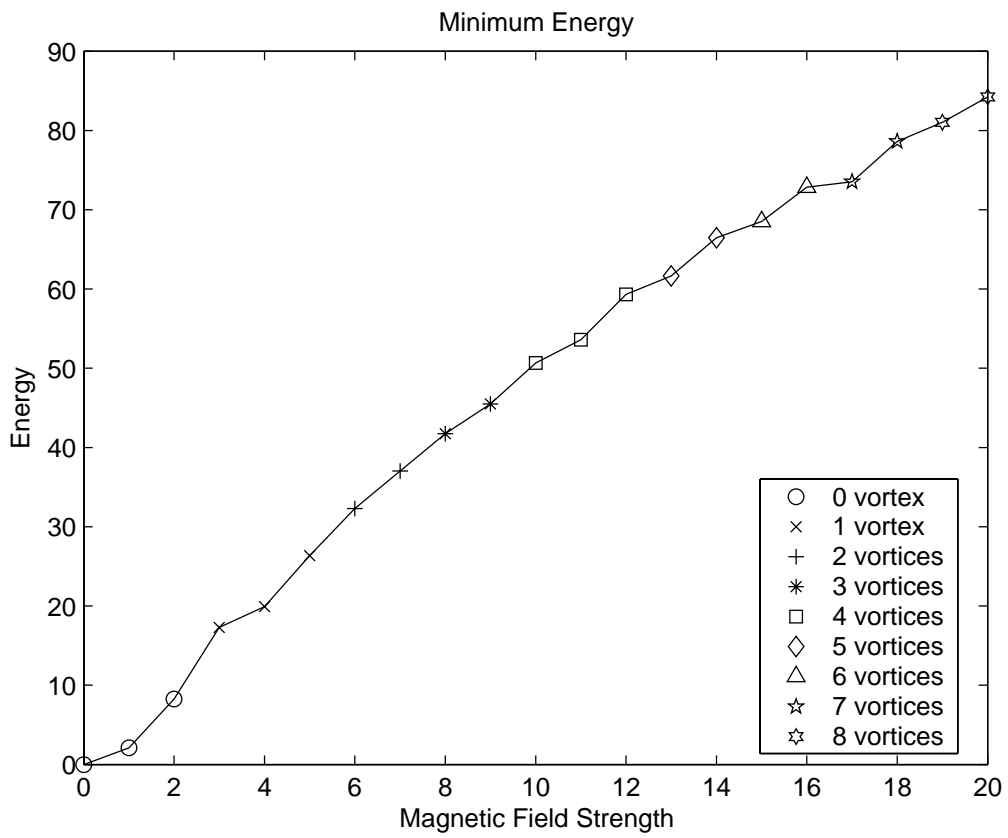

FiguRE 7. Minimum energy $\mathcal{F}^{h}$ vs. magnetics field strength $H$.

form

$$
\begin{cases}\psi(x, y, z)=\frac{x+i y}{\rho}, & |z|>\sqrt{1-\rho^{2}} \\ \psi(x, y, z)=\frac{x+i y}{\sqrt{x^{2}+y^{2}}}, & |z| \leq \sqrt{1-\rho^{2}}\end{cases}
$$

may be used. For small $\rho$, such a function appears to have two vortices at both ends of the poles.

In order to show the convergence of the numerical solution, we compute the solution on grids with $n=162,642,2562,10242$, respectively. For $H=15.0$, a solution representing seven vortices is found on the four different grids, and the computational results are shown in Figure 4 and Table1, The numerical solution obviously demonstrates the convergence as a finer and finer grid is used. Since the exact solution for this case is not known, we take the numerical solution with $n=10242$, denoted by $\psi^{*}$, as a baseline to which we compare the solutions on other grids. Let $F^{*}$ be the corresponding energy of $\psi^{*}$. The relative numerical errors for 

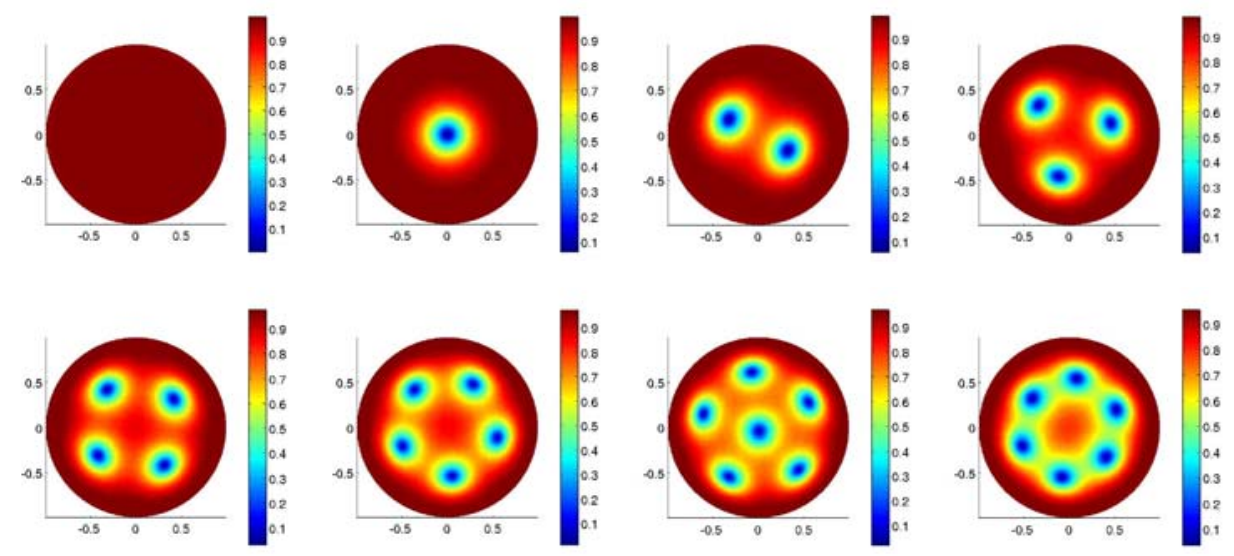

Figure 8. Vortex configurations (density plots of $|\psi|$ ). Top (left to right): vortex free $\left(H=0, \mathcal{F}^{h}=0.0\right), 1$ vortex pair $(H=$ $\left.4, \mathcal{F}^{h}=19.97\right), 2$ pairs $\left(H=7, \mathcal{F}^{h}=37.03\right), 3$ pairs $(H=$

$\left.9, \mathcal{F}^{h}=45.49\right)$; bottom: 4 pairs $\left(H=11, \mathcal{F}^{h}=53.61\right), 5$ pairs

( $\left.H=13, \mathcal{F}^{h}=61.64\right), 6$ pairs $(\mathrm{a})\left(H=15, \mathcal{F}^{h}=68.51\right), 6$ pairs

(b) $\left(H=16, \mathcal{F}^{h}=73.33\right)$.

both $\mathcal{F}^{h}$ and $\left\|\psi^{h}\right\|_{0, \mathbb{S}^{2}}$ are computed and shown in Table1 and the quadratic order convergence is observed.

Figures [5] and 6] contain plots of the corresponding supercurrent $\mathbf{J}_{s}$ computed on the $n=10242$ grid by the discrete gauge invariant gradient recovery scheme and the corrected magnetic field $\mathbf{H}$ with the shell thickness $\delta=0.1$.

Normally, a solution branch is found by path-following with respect to the parameter $H$. Jumping onto other solution branches may occur for some critical values of $H$, then new branches are probed. Repeating such processes, various solution branches become known and some of the corresponding configurations are given in Figure 8 (top view). The corresponding energy values are computed for each solution. For various values of the applied magnetic field $H$, only particular vortex configurations correspond to global minimizers of free energy. Global minimum energy values $\mathcal{F}^{h}$ and corresponding vortex configurations are shown in Figure 7 and Table 2 Note that there are two topologically different vortex configurations all with six vortex pairs (top view) with energy values very close to each other. Nevertheless, the configuration with a vortex pair in the center has a slightly smaller energy value.

\section{Conclusion}

The recently developed spherical centroidal Voronoi tessellations are applied here to the numerical solution of a nonlinear problem arising from the study of the vortex phenomenon in superconductivity, namely, a reduced Ginzburg-Landau model.

In our work, the approximation of the Ginzburg-Landau model for a superconducting hollow sphere based on the SCVT enjoy many interesting mathematical and physical properties, ranging from the maximum principle to gauge invariance 
and high order resolution. They also utilize the properties of the SCVT to provide effective computation of the various solution branches and vortex nucleation patterns on the spheres. Though standard gauge invariant finite difference methods have been well studied and used on tensor based cartesian grids, for spherical geometry, however, the finite volume scheme on the spherical Voronoi tessellations presented here seems to be the only viable alternative for preserving discrete gauge invariance.

For the purpose of illustration, a constant magnetic applied field is considered. The effects of nonconstant fields and the variation in the thickness of the spherical shell have not been investigated. Other more complicated models such as full three-dimensional Ginzburg-Landau models have not been considered either. More detailed studies of the vortex state for superconducting hollow spheres will be made [18 and compared with existing studies for other geometries such as thin films. Nevertheless, the analysis and the numerical simulations presented here clearly demonstrate the effectiveness of the SCVT based numerical discretization methods even for nonlinear problems, especially when the physical and mathematical structures of the physical problem can be utilized. It is expected that SCVT will also find other applications related to the computation of physical models on spheres, for instance, in the simulation of shallow water models and global circulation models.

\section{ACKNOWLEDGMENT}

The authors wish to thank the referee for very helpful comments and suggestions.

\section{REFERENCES}

[1] S. Adler And T. Piran, Relaxation methods for gauge field equilibrium equations, Rev. Mod. Phys., 56 , pp. 1-40, 1984. MR85c:81022

[2] A. Aftalion and Q. Du, The bifurcation diagram for the Ginzburg-Landau system for superconductivity, Physica D, 163, pp. 94-105, 2001. MR2003d:82125

[3] B. Baelus and F. Peeters, Dependence of the vortex configuration on the geometry of mesoscopic flat samples, Phys. Rev. B, 65, 104515, 2002.

[4] B. Baelus, F. Peeters and V. Schweigert, Saddle-point states and energy barriers for vortex entrance and exit in superconducting disks and rings, Phys. Rev. B, 63, 144517, 2001.

[5] S. Chapman, Q. Du and M. Gunzburger, A variable thickness thin film model for superconductivity, Z. Angew. Math. Phys., 47, pp. 410-431 1995. MR2003d:82125

[6] S. Chapman, Q. Du And M. Gunzburger, A Ginzburg-Landau model for superconducting shells, preprint.

[7] S. Chapman, D. Heron, The Motion of Superconducting Vortices in Thin Films of Varying Thickness, SIAM J. Applied Mathematics, 58, pp. 1808-1825, 1998. MR99k:82077

[8] Z. Chen And S. DAI, Adaptive Galerkin method with error control for a dynamical GinzburgLandau model in superconductivity SIAM J. Numer. Anal., 38, pp. 1961-1985, 2001. MR 2002g:65114

[9] M. Coffey, London model for the levitation force between a horizontally oriented point magnetic dipole and superconducting sphere, Phys. Rev. B, 65, 214524, 2002.

[10] S. Ding AND Q. Du, Critical magnetic field and asymptotic behavior for superconducting thin films, SIAM J. Mathematical Analysis, 34, pp. 239-256, 2002. MR2004c:35398

[11] M. Dodgson And M.A. Moore, Vortices in a thin-film superconductor with a spherical geometry, Phys. Rev. B, 55, pp. 3816-3831, 1997.

[12] Q. Du, Discrete gauge invariant approximations of a time-dependent Ginzburg-Landau model of superconductivity, Math. Comp., 67, pp. 965-986, 1998. MR 98m:820774

[13] Q. Du, M. Gunzburger, And L. Ju, Constrained centroidal Voronoi tessellations on general surfaces, SIAM J. Sci. Comput., 24, pp.1488-1506, 2003. MR.2004d:65155 
[14] Q. Du, M. Gunzburger and L. Ju, Voronoi-based finite volume methods, optimal Voronoi meshes, and PDEs on the sphere, Comp. Meth. Appl. Mech. Engrg., 192, pp. 779-802, 2003. MR 2004g:65142

[15] Q. Du, M. Gunzburger M. and J. Peterson, Analysis and approximation of the GinzburgLandau model of superconductivity, SIAM Review, 34, pp. 54-81, 1992. MR93g:82109

[16] Q. Du, M. Gunzburger and J. Peterson, Computational simulations of type-II superconductivity including pinning mechanisms, Phys. Rev. B, 51, pp. 16194-16203, 1995.

[17] Q. Du And L. Ju, Finite volume methods on spheres and spherical centroidal Voronoi tessellations, IMA preprint, No. 1918, 2003.

[18] Q. DU AND L. JU, Numerical simulation of the quantized vortices on a thin superconducting hollow sphere, to appear in J. Computational Phys., 2004.

[19] Q. Du AND F. Lin, Ginzburg-Landau vortices: dynamics, pinning and hysteresis, SIAM J. Math. Anal., 28, pp. 1265-1293, 1999. MR99c:82075

[20] Q. Du, R. Nicolaides And X. Wu, Analysis and convergence of a covolume approximation of the Ginzburg-Landau models of superconductivity, SIAM J. Numer. Anal., 35, pp. 10491072, 1998. MR99e:65178

[21] W. E., Dynamics of vortices in Ginzburg-Landau theories with applications to superconductivity, Phys. D, 77, pp. 383-404, 1994. MR95g:82097

[22] V. Fomin, V. Misko, J. Devreese and V. Moshchalkov, Superconducting mesoscopic square loop, Phys. Rev. B, 58, pp. 11703-11715, 1998. MR]

[23] W. Gropp, H. Kaper, G. Leaf, D. Levine, M. Palumbo, and V. Vinokur, Numerical simulation of vortex dynamics in type-II superconductors, J. Comp. Phys., 123, pp. 254-266, 1996.

[24] E. HeBey, Sobolev Spaces on Riemannian Manifolds, Springer, Berlin, 1991. MR 98k:46049

[25] B. IanotTa, Music of the Spheres, New Scientist, 31, pp.28-31, 1996.

[26] H. Jadallah, J. Rubinstein, and P. Sternberg, Phase Transition Curves for Mesoscopic Superconducting Samples, Phys. Rev. Lett, 82, pp. 2935, 1999.

[27] E. Polak, Computational Methods in Optimization, Academic Press, New York, 1971. MR $43: 8222$

[28] R. RENKA; Algorithm 772: STRIPACK: Delaunay triangulation and Voronoi diagrams on the surface of a sphere, ACM Trans. Math. Soft., 23, pp. 416-434, 1997.

[29] B. Richter and R. Warburton, A new generation of superconducting gravimeters, in Proceedings of the 13th Intern. Sym. on Earth Tides, Brussel, pp. 545-556, 1998, Série Géophysique, Royal Observatory of Belgium.

[30] V. Schweigert, F. Peeters and P. Deo, Vortex Phase Diagram for Mesoscopic Superconducting Disks, Phys. Rev. Lett, 81, pp. 2783-2786, 1998.

[31] R. Tao, X. Zhang, X. Tang, and P. Anderson, Formation of High Temperature Superconducting Balls, Phys. Rev. Lett, 83, pp. 5575-5578, 1999.

[32] B. Tent, D. Qu, D. Shi, W. Bresser, P. Boolchand and Z. Cai, Angle dependence of magnetization in a single-domain $\mathrm{YBa}_{2} \mathrm{Cu}_{3} \mathrm{O}_{x}$ sphere, Phys. Rev. B, 58, 11761, 1998.

[33] M. Tinkham, Introduction to Superconductivity, 2nd ed., McGraw-Hill, New York, 1994.

[34] J. Yeo and M. Moore, Non-integer flux quanta for a spherical superconductor, Phys. Rev. B, 58, pp. 10785-10789, 1998.

[35] Y. Xiao, S. Buchman, G Keiser, B. Muhlfelder, J. Turneaure, and C. Wu, Magnetic flux distribution on a spherical superconducting shell, Physica B, 194, pp.65-66, 1994.

Department of Mathematics, Pennsylvania State University, University Park, PennSYLVANIA 16802

E-mail address: qdu@math.psu.edu

Institute for Mathematics and its Applications, University of Minnesota, MinneapoLis, Minnesota 55455

E-mail address: ju@ima.umn.edu 